\title{
Fluctuating hydrodynamics for dilute granular gases
}

\author{
J. Javier Brey, ${ }^{1}$ P. Maynar, ${ }^{1,2}$ and M.I. García de Soria ${ }^{1,3}$ \\ ${ }^{1}$ Física Teórica, Universidad de Sevilla, \\ Apartado de Correos 1065, E-41080, Sevilla, Spain \\ ${ }^{2}$ Laboratoire de Physique Théorique (CNRS UMR 8627), \\ Bâtiment 210, Université Paris-Sud, 91405 Orsay Cedex, France \\ ${ }^{3}$ LPTMS (CNRS UMR 8626), Université Paris-Sud, Orsay Cedex, F-91405, France
}

(Dated: November 3, 2018)

\begin{abstract}
Starting from the kinetic equations for the fluctuations and correlations of a dilute gas of inelastic hard spheres or disks, a Boltzmann-Langevin equation for the one-particle distribution function of the homogeneous cooling state is constructed. This equation is the linear Boltzmann equation with a fluctuating white noise term. Balance equations for the fluctuating hydrodynamic fields are derived. New fluctuating forces appear as compared with the elastic limit. The particular case of the transverse velocity field is investigated in detail. Its fluctuations can be described by means of a Langevin equation, but exhibiting two main differences with the Landau-Lifshitz theory: the noise is not white, and its second moment is not determined by the shear viscosity. This shows that the fluctuation-dissipation relations for molecular fluids do not straightforwardly carry over to inelastic gases. The theoretical predictions are shown to be in good agreement with molecular dynamics simulation results.
\end{abstract}

PACS numbers: 45.70.Mg, 45.70.Qj, 47.20.Ky 


\section{INTRODUCTION}

The (modified) nonlinear Boltzmann equation for the one particle distribution function provides an accurate description of transport phenomena in a low density gas of inelastic hard spheres or disks [1, 2, 3, 4, 5]. These particles are often used to model granular fluids [6], specially in the rapid flow regime [7]. The Boltzmann equation does not provide any direct information about correlations and fluctuations in the gas, other that the particle velocity moments. Nevertheless, methods used in the derivation of the Boltzmann equation have been extended to obtain kinetic equations for the equal and different time correlations, in the same low density approximation. The general idea is that in order to obtain these equations the needed approximations are the same as those used to derive the Boltzmann equation itself.

One of the earliest and physically more transparent methods to study fluctuations is that of Langevin equations. Almost 40 years ago, in a seminal paper Bixon and Zwanzig [8] showed how a Boltzmann-Langevin equation could be constructed by generalizing the reasonings leading to the Boltzmann equation for molecular gases. The latter describes the behavior of the average value of the one-particle distribution function, while the former incorporates the effects of the fluctuations. As the authors indicated themselves in the paper, the derivation was based on physical intuition and analogy. A more systematic derivation of the same result, starting from first principles, was given in ref. [9].

A second approach to the study of correlations in dilute gases makes use of functional analysis. Its more general result is a kinetic equation for a generating functional at low density, from which all multi-point correlations can be obtained by functional differentiation [10]. A closely related general scheme for the study of correlations is the hierarchical method [11, 12]. The starting point are hierarchies of coupled equations for the time distribution functions describing the fluctuations and correlations. Then, the hierarchies are closed by using the same kind of approximations as needed to derive the kinetic equation, i.e. the Boltzmann equation in the case of dilute gases. This method has been recently extended to describe fluctuations and correlations of dilute inelastic gases in their simplest state, the homogeneous cooling state (HCS) [13]. As an application, the fluctuations of the total energy were studied and a good agreement between theory and simulation results was found [13, 14]. 
One of the aims of this paper is to translate the above formalism in terms of kinetic equations for the correlation functions into a Langevin equation formulation, i.e. to extend the fluctuating Boltzmann equation to the case of inelastic hard spheres or disks. The relationship between kinetic equations and the fluctuating Boltzmann equation has been analyzed in detail in molecular gases [12, 15]. One advantage of the Langevin formulation is that it is closer to the fluctuating hydrodynamic equations. Actually, the fluctuating Boltzmann equation for molecular systems has been shown [8, 16, 17] to lead to the same Langevin equations for the hydrodynamic fields as obtained by Landau and Lifshitz [18] using thermodynamic fluctuation theory. The noise terms in these equations are assumed to be white with second moments determined by the Navier-Stokes transport coefficients of the fluid. Their expressions are known as fluctuation-dissipation relations of the second kind [19].

The derivation of fluctuating hydrodynamic equations from the fluctuating Boltzmann equation for inelastic hard particles, will be also addressed here. Attention will be focussed on a particular state, the HCS, and on a specific hydrodynamic field, the transverse component of the velocity. The main conclusion will be that the fluctuation-dissipation relation for elastic gases can not be directly extrapolated to inelastic ones, but it needs to be significantly modified. The second moment of the noise is not determined by the Navier-Stokes shear viscosity. Moreover, the noise can not be assumed to be white. These theoretical predictions are in qualitative and quantitative agreement with molecular dynamics simulation results.

The consideration of the HCS does not imply by itself that the results obtained here are not relevant for other states more accesible experimentally. The HCS plays for inelastic gases a role similar to the equilibrium state for molecular gases. In the case of molecular systems, the expressions of the transport coefficients obtained by linearizing around equilibrium are the same as those appearing in the nonlinear Navier-Stokes equations as predicted by the Chapman-Enskog method and successfully used in many far from equilibrium problems [20]. Also, the fluctuation-dissipation relations derived for near-local-equilibrium states in the original Landau and Lifshitz theory have proven to be accurate for many other hydrodynamic states [21]. For dilute gases composed of inelastic hard particles, the equivalence between the transport coefficients obtained by linear perturbations of the HCS and by applying the Chapman-Enskog procedure has also been established [22]. Something similar might be expected for the fluctuations and correlations. 
In the system being considered here, the particles move freely and independently between consecutive collisions. More specifically, they are not coupled to any external energy source or thermal bath, contrary to the driven granular gas models. For these models, the linear response to an external perturbation [23] as well as the validity of the Einstein relation 24] have been investigated by numerical simulations, and some empirical models have been proposed. It is not evident a direct relation between the free model considered here and the above driven models.

The plan of the paper is as follows. In Sec. II, the kinetic equations for the one-time and two-time correlation functions of a dilute gas in the HCS derived in ref. [13] are shortly reviewed. These equations are translated into an equivalent Boltzmann-Langevin equation for the one particle distribution function in Sec. III] When written in the appropriate variables, this equation is the linear Boltzmann equation to which a fluctuating force term is added, similarly to what happens in molecular elastic gases. An expression for the second moment of the fluctuating force in terms of the collisional Boltzmann kernel is derived. In Sec. IV, the fluctuating hydrodynamic fields are defined, and balance equations for them are obtained from the Boltzmann-Langevin equation. They involve formal expressions for the fluctuating pressure tensor, the fluctuating heat flux, and the fluctuating cooling rate. In addition, an intrinsically inelastic fluctuating force shows up in the equation for the energy.

To get a closed description for the hydrodynamic fluctuations, expressions for the heat flux, the pressure tensor, and the cooling rate in terms of the fluctuating hydrodynamic fields are needed. This can be accomplished by means of the Chapman-Enskog procedure. Here only the case of the transverse component of the velocity field will be considered. As a consequence, only the expression for the non-diagonal elements of the pressure tensor is required. This is computed in Sec. $\mathrm{V}$. The final result is a Langevin equation, that is the linear macroscopic equation for the transverse velocity field plus a fluctuating force term. Therefore, the structure is similar to what one could expect by extrapolating from the corresponding equation for molecular systems [25]. Nevertheless, the noise term is not white and its second moment is not given by the usual fluctuation-dissipation relation. It is verified that the obtained theoretical predictions are in good agreement with molecular dynamics simulation results. Section VII contains some general comments and conclusions. Finally, the appendixes provide some details of the calculations needed to derive the results presented in the bulk of the paper. 


\section{KINETIC EQUATIONS FOR THE HOMOGENEOUS COOLING STATE}

The system considered is a dilute gas of $N$ smooth inelastic hard spheres $(d=3)$ or disks $(d=2)$ of mass $m$ and diameter $\sigma$. The position and velocity of the $i$ th particle at time $t$ will be denote by $\boldsymbol{R}_{i}(t)$ and $\boldsymbol{V}_{i}(t)$, respectively. The effect of a collision between particles $i$ and $j$ is to instantaneously modify their velocities according to the rule

$$
\begin{aligned}
\boldsymbol{V}_{i} & \rightarrow \boldsymbol{V}_{i}^{\prime}=\boldsymbol{V}_{i}-\frac{1+\alpha}{2}\left(\widehat{\boldsymbol{\sigma}} \cdot \boldsymbol{V}_{i j}\right) \widehat{\boldsymbol{\sigma}}, \\
\boldsymbol{V}_{j} \rightarrow \boldsymbol{V}_{j}^{\prime} & =\boldsymbol{V}_{j}+\frac{1+\alpha}{2}\left(\widehat{\boldsymbol{\sigma}} \cdot \boldsymbol{V}_{i j}\right) \widehat{\boldsymbol{\sigma}},
\end{aligned}
$$

where $\boldsymbol{V}_{i j}=\boldsymbol{V}_{i}-\boldsymbol{V}_{j}$ is the relative velocity, $\widehat{\boldsymbol{\sigma}}$ is the unit vector pointing from the center of particle $j$ to the center of particle $i$ at contact, and $\alpha$ is the coefficient of normal restitution. It is defined in the interval $0<\alpha \leq 1$ and it will considered here as constant, independent of the velocities of the particles involved in the collision. A more realistic modeling of granular gases would require to consider a velocity dependent restitution coefficient [5].

Given a trajectory of the system, one-point and two-point microscopic densities in phase space at time $t$ are defined by

$$
F_{1}\left(x_{1}, t\right)=\sum_{j=1}^{N} \delta\left[x_{1}-X_{j}(t)\right]
$$

and

$$
F_{2}\left(x_{1}, x_{2}, t\right)=\sum_{i}^{N} \sum_{j \neq i}^{N} \delta\left[x_{1}-X_{i}(t)\right] \delta\left[x_{2}-X_{j}(t)\right],
$$

respectively. Here $X_{i}(t) \equiv\left\{\boldsymbol{R}_{i}(t), \boldsymbol{V}_{i}(t)\right\}$, while the $x_{i} \equiv\left\{\boldsymbol{r}_{i}, \boldsymbol{v}_{i}\right\}$ are field variables referring to the one-particle phase space $\left(\mu\right.$ space). The density $F_{1}\left(x_{1}, t\right)$ obeys the equation $[11,13]$

$$
\left[\frac{\partial}{\partial t}+\boldsymbol{v}_{1} \cdot \frac{\partial}{\partial \boldsymbol{r}_{1}}\right] F_{1}\left(x_{1}, t\right)=\int d x_{2} \bar{T}\left(x_{1}, x_{2}\right) F_{2}\left(x_{1}, x_{2}, t\right)
$$

with

$$
\bar{T}\left(x_{i}, x_{j}\right)=\sigma^{d-1} \int d \widehat{\boldsymbol{\sigma}} \Theta\left(\boldsymbol{v}_{i j} \cdot \widehat{\boldsymbol{\sigma}}\right)\left|\boldsymbol{v}_{i j} \cdot \widehat{\boldsymbol{\sigma}}\right|\left[\alpha^{-2} \delta\left(\boldsymbol{r}_{i j}-\boldsymbol{\sigma}\right) b_{\boldsymbol{\sigma}}^{-1}\left(\boldsymbol{v}_{i}, \boldsymbol{v}_{j}\right)-\delta\left(\boldsymbol{r}_{i j}+\boldsymbol{\sigma}\right)\right],
$$

where $d \widehat{\boldsymbol{\sigma}}$ is the solid angle element for $\widehat{\boldsymbol{\sigma}} \equiv \boldsymbol{\sigma} / \sigma, \boldsymbol{r}_{12} \equiv \boldsymbol{r}_{1}-\boldsymbol{r}_{2}, \Theta$ is the Heaviside step function, and $b_{\boldsymbol{\sigma}}^{-1}\left(\boldsymbol{v}_{1}, \boldsymbol{v}_{2}\right)$ is an operator replacing all the functions of $\boldsymbol{v}_{1}$ and $\boldsymbol{v}_{2}$ to its right by the same functions of the precollisional values $\boldsymbol{v}_{1}^{*}$ and $\boldsymbol{v}_{2}^{*}$ given by

$$
\begin{gathered}
\boldsymbol{v}_{1}^{*} \equiv b_{\boldsymbol{\sigma}}^{-1} \boldsymbol{v}_{1}=\boldsymbol{v}_{1}-\frac{1+\alpha}{2 \alpha}\left(\widehat{\boldsymbol{\sigma}} \cdot \boldsymbol{v}_{12}\right) \widehat{\boldsymbol{\sigma}} \\
\boldsymbol{v}_{2}^{*} \equiv b_{\boldsymbol{\sigma}}^{-1} \boldsymbol{v}_{2}=\boldsymbol{v}_{2}+\frac{1+\alpha}{2 \alpha}\left(\widehat{\boldsymbol{\sigma}} \cdot \boldsymbol{v}_{12}\right) \widehat{\boldsymbol{\sigma}} .
\end{gathered}
$$


It is seen that Eq. (4) for $F_{1}$ involves the two particle density $F_{2}$. Actually, it is the first equation of an infinity hierarchy [13].

The averages of $F_{1}\left(x_{1}, t\right)$ and $F_{2}\left(x_{1}, x_{2}, t\right)$ over the initial probability distribution of the system $\rho(\Gamma, 0), \Gamma \equiv\left\{X_{1}, \ldots, X_{N}\right\}$, are the usual one-particle and two-particle distribution functions,

$$
f_{1}\left(x_{1}, t\right)=\left\langle F_{1}\left(x_{1}, t\right)\right\rangle, \quad f_{2}\left(x_{1}, x_{2}, t\right)=\left\langle F_{2}\left(x_{1}, x_{2}, t\right)\right\rangle
$$

where the notation

$$
\langle G\rangle \equiv \int d \Gamma G(\Gamma) \rho(\Gamma, 0)
$$

has been employed. Two-time reduced distribution functions can also defined from the microscopic densities and the initial probability distribution. The simplest one is the twoparticle two-time distribution function,

$$
f_{1,1}\left(x_{1}, t ; x_{1}^{\prime}, t^{\prime}\right)=\left\langle F_{1}\left(x_{1}, t\right) F_{1}\left(x_{1}^{\prime}, t^{\prime}\right)\right\rangle
$$

From the definitions in Eqs. (7) and (9) it follows that

$$
f_{1,1}\left(x_{1}, t ; x_{1}^{\prime}, t\right)=\delta\left(x_{1}-x_{1}^{\prime}\right) f_{1}\left(x_{1}, t\right)+f_{2}\left(x_{1}, x_{1}^{\prime}, t\right) .
$$

It is convenient to introduce one-time and two-time correlation functions by

$$
g_{2}\left(x_{1}, x_{2}, t\right) \equiv f_{2}\left(x_{1}, x_{2}, t\right)-f_{1}\left(x_{1}, t\right) f_{1}\left(x_{2}, t\right)
$$

and

$$
h_{1,1}\left(x_{1}, t ; x_{1}^{\prime}, t^{\prime}\right) \equiv f_{1,1}\left(x_{1}, t ; x_{1}^{\prime}, t^{\prime}\right)-f_{1}\left(x_{1}, t\right) f_{1}\left(x_{1}^{\prime} ; t^{\prime}\right)
$$

respectively. Equation (10) translates into

$$
h_{1,1}\left(x_{1}, t ; x_{1}^{\prime}, t\right)=\delta\left(x_{1}-x_{1}^{\prime}\right) f_{1}\left(x_{1}, t\right)+g_{2}\left(x_{1}, x_{1}^{\prime}, t\right)
$$

In the low density limit, a closed set of kinetic equations for $f_{1}, g_{2}$, and $h_{1,1}$ can be derived [13] by extending the methods developed for molecular gases [12]. They can be used to analyze the average properties as well as correlations and fluctuations in arbitrary states of a dilute granular gas. Here attention will be restricted to a particular state of a freely evolving granular gas, the so-called homogeneous cooling state (HCS) [26]. Macroscopically, it is characterized by a uniform number of particles density $n$, a vanishing velocity field, 
and a uniform time-dependent temperature $T(t)$. It is further defined by the one-particle distribution function having the scaled form [1]

$$
f(\boldsymbol{v}, t)=n v_{0}^{-d}(t) \chi(c)
$$

where

$$
v_{0}(t) \equiv\left[\frac{2 T(t)}{m}\right]^{1 / 2}
$$

is a thermal velocity and $\chi(c)$ is an isotropic function of the scaled velocity $\boldsymbol{c} \equiv \boldsymbol{v} / v_{0}(t)$. The distribution $\chi(c)$ and the granular temperature $T(t)$ are specified by the pair of coupled equations

$$
\begin{gathered}
\frac{\partial T}{\partial s}=-\zeta_{0} T(s) \\
\frac{\zeta_{0}}{2} \frac{\partial}{\partial \boldsymbol{c}} \cdot(\boldsymbol{c} \chi)=J_{c}[\boldsymbol{c} \mid \chi] .
\end{gathered}
$$

In the above expressions,

$$
\zeta_{0}=\frac{\left(1-\alpha^{2}\right) \pi^{\frac{d-1}{2}}}{2 \Gamma\left(\frac{d+3}{2}\right) d} \int d \boldsymbol{c}_{1} \int d \boldsymbol{c}_{2} c_{12}^{3} \chi\left(c_{1}\right) \chi\left(c_{2}\right)
$$

is the dimensionless cooling rate in the time scale $s$ defined by

$$
s \equiv \int_{0}^{t} d t_{1} \frac{v_{0}\left(t_{1}\right)}{\lambda}
$$

with $\lambda \equiv\left(n \sigma^{d-1}\right)^{-1}$, and $J_{c}[\boldsymbol{c} \mid \chi]$ is the inelastic Boltzmann collision term. Its explicit form is

$$
\begin{gathered}
J_{c}[\boldsymbol{c} \mid \chi]=\int d \boldsymbol{c}_{1} \bar{T}_{0}\left(\boldsymbol{c}, \boldsymbol{c}_{1}\right) \chi(c) \chi\left(c_{1}\right), \\
\bar{T}_{0}\left(\boldsymbol{c}, \boldsymbol{c}_{1}\right)=\int d \widehat{\boldsymbol{\sigma}} \Theta\left[\left(\boldsymbol{c}-\boldsymbol{c}_{1}\right) \cdot \widehat{\boldsymbol{\sigma}}\right]\left(\boldsymbol{c}-\boldsymbol{c}_{1}\right) \cdot \widehat{\boldsymbol{\sigma}}\left[\alpha^{-2} b_{\boldsymbol{\sigma}}^{-1}\left(\boldsymbol{c}, \boldsymbol{c}_{1}\right)-1\right] .
\end{gathered}
$$

The variable $s$ defined in Eq. (19) is proportional to the accumulated number of collisions per particle. For thermal velocities, i.e values of $c$ of the order of unity, a good approximation to the solution of Eqs. (16) and (17) is provided by the first Sonine approximation, in which [1, 27]

$$
\chi(c)=\frac{e^{-c^{2}}}{\pi^{d / 2}}\left[1+a_{2}(\alpha) S^{(2)}\left(c^{2}\right)\right]
$$

with

$$
S^{(2)}\left(c^{2}\right)=\frac{c^{4}}{4}-\frac{d+2}{2} c^{2}+\frac{d(d+2)}{8}
$$


and

$$
a_{2}(\alpha)=\frac{16(1-\alpha)\left(1-2 \alpha^{2}\right)}{9+24 d+(8 d-41) \alpha+30 \alpha^{2}-30 \alpha^{3}} .
$$

In the same approximation

$$
\zeta_{0}=\frac{\sqrt{2} \pi^{(d-1) / 2}\left(1-\alpha^{2}\right)}{\Gamma(d / 2) d}\left[1+\frac{3 a_{2}(\alpha)}{16}\right] .
$$

A numerically exact solution of Eqs. (30) and (17) has been recently reported in [28]. The two-particle one-time correlation function of the HCS is assumed to have also a scaled form [13]

$$
g_{2}\left(\boldsymbol{r}_{12}, \boldsymbol{v}_{1}, \boldsymbol{v}_{2}, t\right)=n \lambda^{-d} v_{0}^{-2 d}(t) \widetilde{g}\left(\boldsymbol{l}_{12}, \boldsymbol{c}_{1}, \boldsymbol{c}_{2}\right),
$$

where the scaled length scale $\boldsymbol{l} \equiv \boldsymbol{r} / \lambda$ has been introduced. The dimensionless correlation $\widetilde{g}$ does not depend on $s$ and obeys the equation

$$
\left[\boldsymbol{c}_{12} \cdot \frac{\partial}{\partial \boldsymbol{l}_{12}}-\Lambda\left(\boldsymbol{c}_{1}\right)-\Lambda\left(\boldsymbol{c}_{2}\right)\right] \tilde{g}\left(\boldsymbol{l}_{12}, \boldsymbol{c}_{1}, \boldsymbol{c}_{2}\right)=\delta\left(\boldsymbol{l}_{12}\right) \bar{T}_{0}\left(\boldsymbol{c}_{1}, \boldsymbol{c}_{2}\right) \chi\left(c_{1}\right) \chi\left(c_{2}\right),
$$

where $\Lambda\left(\boldsymbol{c}_{i}\right)$ is the linearized Boltzmann collision operator [29],

$$
\Lambda\left(\boldsymbol{c}_{i}\right) \equiv \int d \boldsymbol{c}_{3} \bar{T}_{0}\left(\boldsymbol{c}_{i}, \boldsymbol{c}_{3}\right)\left(1+P_{i 3}\right) \chi\left(c_{3}\right)-\frac{\zeta_{0}}{2} \frac{\partial}{\partial \boldsymbol{c}_{i}} \cdot \boldsymbol{c}_{i}
$$

The operator $P_{i j}$ interchanges the labels of particles $i$ and $j$ of the quantities to its right. For the two-particle two-time correlation function the scaling reads [13]

$$
h_{1,1}\left(x_{1}, t ; x_{1}^{\prime}, t^{\prime}\right)=n \lambda^{-d} v_{0}^{-d}(t) v_{0}^{-d}\left(t^{\prime}\right) \widetilde{h}\left(\boldsymbol{l}_{1}-\boldsymbol{l}_{1}^{\prime}, \boldsymbol{c}_{1}, s-s^{\prime} ; \boldsymbol{c}_{1}^{\prime}\right)
$$

and the kinetic equation is

$$
\left[\frac{\partial}{\partial s}+\boldsymbol{c}_{1} \cdot \frac{\partial}{\partial \boldsymbol{l}_{1}}-\Lambda\left(\boldsymbol{c}_{1}\right)\right] \tilde{h}\left(\boldsymbol{l}_{1}-\boldsymbol{l}_{1}^{\prime}, \boldsymbol{c}_{1}, s-s^{\prime} ; \boldsymbol{c}_{1}^{\prime}\right)=0,
$$

valid for $s>s^{\prime}>0$. The initial condition for this equation is

$$
\begin{aligned}
\widetilde{h}\left(\boldsymbol{l}_{1}-\boldsymbol{l}_{1}^{\prime}, \boldsymbol{c}_{1}, 0 ; \boldsymbol{c}_{1}^{\prime}\right) & \equiv \tilde{h}_{1,1}\left(\boldsymbol{l}_{1}-\boldsymbol{l}_{1}^{\prime}, \boldsymbol{c}_{1} ; \boldsymbol{c}_{1}^{\prime}\right) \\
& =\tilde{g}\left(\boldsymbol{l}_{1}-\boldsymbol{l}_{1}^{\prime}, \boldsymbol{c}_{1}, \boldsymbol{c}_{1}^{\prime}\right)+\delta\left(\boldsymbol{c}_{1}-\boldsymbol{c}_{1}^{\prime}\right) \delta\left(\boldsymbol{l}_{1}-\boldsymbol{l}_{1}^{\prime}\right) \chi\left(c_{1}\right)
\end{aligned}
$$

An equation for this distribution follows from Eqs. (17) and (27),

$$
\left[\boldsymbol{c}_{1} \cdot \frac{\partial}{\partial \boldsymbol{l}_{1}}+\boldsymbol{c}_{1}^{\prime} \cdot \frac{\partial}{\partial \boldsymbol{l}_{1}^{\prime}}-\Lambda\left(\boldsymbol{c}_{1}\right)-\Lambda\left(\boldsymbol{c}_{1}^{\prime}\right)\right] \widetilde{h}\left(\boldsymbol{l}_{1}-\boldsymbol{l}_{1}^{\prime}, \boldsymbol{c}_{1} ; \boldsymbol{c}_{1}^{\prime}\right)=\delta\left(\boldsymbol{l}_{1}-\boldsymbol{l}_{1}^{\prime}\right) \widetilde{\Gamma}\left(\boldsymbol{c}_{1}, \boldsymbol{c}_{1}^{\prime}\right)
$$


with

$$
\widetilde{\Gamma}\left(\boldsymbol{c}_{1}, \boldsymbol{c}_{1}^{\prime}\right)=-\left[\Lambda\left(\boldsymbol{c}_{1}\right)+\Lambda\left(\boldsymbol{c}_{1}^{\prime}\right)\right] \delta\left(\boldsymbol{c}_{1}-\boldsymbol{c}_{1}^{\prime}\right) \chi\left(c_{1}\right)+\bar{T}_{0}\left(\boldsymbol{c}_{1}, \boldsymbol{c}_{1}^{\prime}\right) \chi\left(c_{1}\right) \chi\left(c_{1}^{\prime}\right)
$$

Equations (27) and (30) describe the correlations between fluctuations in the HCS. They become closed once the solution to Eqs. (16) and (17) is known. In the next section, an alternative and consistent description to that provided by these kinetic equations will be developed.

\section{FLUCTUATING BOLTZMANN EQUATION AROUND THE HCS}

Equation (41) is an exact consequence of the dynamical equations governing the motion of the particles. The aim of this section is to approximate it in such a way that give a closed description of the effective dynamics of a dilute granular gas in the HCS. To do so, the spatial separation between the centers of colliding particles will be neglected in the operator $\bar{T}\left(x_{1}, x_{2}\right)$, and $F_{2}\left(x_{1}, x_{2}, t\right)$ will be approximated by an effective (Boltzmann) two-particle

phase space density at the mesoscopic level $F_{2}^{B}\left(x_{1}, x_{2}, t\right)$. Moreover, the dimensionless time scale $s$ and length scale $\boldsymbol{l}$ introduced in the previous section will be used. Then, Eq. (4) becomes

$$
\left(\frac{\partial}{\partial s}+\frac{\zeta_{0}}{2} \frac{\partial}{\partial \boldsymbol{c}_{1}} \cdot \boldsymbol{c}_{1}+\boldsymbol{c}_{1} \cdot \frac{\partial}{\partial \boldsymbol{l}_{1}}\right) \widetilde{F}_{1}\left(\boldsymbol{l}_{1}, \boldsymbol{c}_{1}, s\right)=\int d \boldsymbol{c}_{2} \bar{T}_{0}\left(\boldsymbol{c}_{1}, \boldsymbol{c}_{2}\right) \widetilde{F}_{2}^{B}\left(\boldsymbol{l}_{1}, \boldsymbol{c}_{1}, \boldsymbol{l}_{1}, \boldsymbol{c}_{2}, s\right),
$$

where dimensionless phase space densities have been defined by

$$
\begin{gathered}
\widetilde{F}_{1}\left(\boldsymbol{l}_{1}, \boldsymbol{c}_{1}, s\right)=n^{-1} v_{0}^{d}(t) F_{1}\left(x_{1}, t\right), \\
\widetilde{F}_{2}^{B}\left(\boldsymbol{l}_{1}, \boldsymbol{c}_{1}, \boldsymbol{l}_{2}, \boldsymbol{c}_{2}, s\right)=n^{-2} v_{0}^{2 d}(t) F_{2}^{B}\left(x_{1}, x_{2}, t\right) .
\end{gathered}
$$

Comparison of the ensemble average of Eq. (34) with Eq. (17) gives the conditions

$$
\begin{gathered}
\left\langle\widetilde{F}_{1}\left(\boldsymbol{l}_{1}, \boldsymbol{c}_{1}, s\right)\right\rangle_{\mathrm{H}}=\chi\left(c_{1}\right), \\
\int d \boldsymbol{c}_{2} \bar{T}_{0}\left(\boldsymbol{c}_{1}, \boldsymbol{c}_{2}\right)\left\langle\widetilde{F}_{2}^{B}\left(\boldsymbol{l}_{1}, \boldsymbol{c}_{1}, \boldsymbol{l}_{1}, \boldsymbol{c}_{2}, s\right)\right\rangle_{\mathrm{H}}=J_{c}[\boldsymbol{c} \mid \chi] .
\end{gathered}
$$

The subindex $\mathrm{H}$ in the angular brackets indicates that the ensemble average is taken over the probability distribution for the HCS.

The deviation of the microscopic density from its average value is defined by

$$
\delta \widetilde{F}_{1}\left(\boldsymbol{l}_{1}, \boldsymbol{c}_{1}, s\right) \equiv \widetilde{F}_{1}\left(\boldsymbol{l}_{1}, \boldsymbol{c}_{1}, s\right)-\chi\left(c_{1}\right)
$$


An evolution equation for this quantity follows by subtracting Eqs. (34) and (17),

$$
\begin{aligned}
\left(\frac{\partial}{\partial s}\right. & \left.+\frac{\zeta_{0}}{2} \frac{\partial}{\partial \boldsymbol{c}_{1}} \cdot \boldsymbol{c}_{1}+\boldsymbol{c}_{1} \cdot \frac{\partial}{\partial \boldsymbol{l}_{1}}\right) \delta \widetilde{F}_{1}\left(\boldsymbol{l}_{1}, \boldsymbol{c}_{1}, s\right) \\
& =\int d \boldsymbol{c}_{2} \bar{T}_{0}\left(\boldsymbol{c}_{1}, \boldsymbol{c}_{2}\right)\left[\widetilde{F}_{2}^{B}\left(\boldsymbol{l}_{1}, \boldsymbol{c}_{1}, \boldsymbol{l}_{1}, \boldsymbol{c}_{2}, s\right)-\chi\left(c_{1}\right) \chi\left(c_{2}\right)\right] .
\end{aligned}
$$

The structure of this equations suggests to introduce a cluster decomposition for $\widetilde{F}_{2}^{B}$ of the form

$$
\widetilde{F}_{2}^{B}\left(\boldsymbol{l}_{1}, \boldsymbol{c}_{1}, \boldsymbol{l}_{1}, \boldsymbol{c}_{2}, s\right)=\chi\left(c_{1}\right) \chi\left(c_{2}\right)+\chi\left(c_{1}\right) \delta \widetilde{F}_{1}\left(\boldsymbol{l}_{1}, \boldsymbol{c}_{2}, s\right)+\chi\left(c_{2}\right) \delta \widetilde{F}_{1}\left(\boldsymbol{l}_{1}, \boldsymbol{c}_{1}, s\right)+\widetilde{\Phi}_{2}^{B}\left(\boldsymbol{l}_{1}, \boldsymbol{c}_{1}, \boldsymbol{c}_{2}, s\right)
$$

This equation defines the microscopic correlation density $\widetilde{\Phi}_{2}^{B}\left(\boldsymbol{l}_{1}, \boldsymbol{c}_{1}, \boldsymbol{c}_{2}, s\right)$. Substitution of its ensemble average in Eq. (38) yields

$$
\int d \boldsymbol{c}_{2} \bar{T}_{0}\left(\boldsymbol{c}_{1}, \boldsymbol{c}_{2}\right)\left\langle\widetilde{\Phi}_{2}^{B}\left(\boldsymbol{l}_{1}, \boldsymbol{c}_{1}, \boldsymbol{c}_{2}, s\right)\right\rangle_{\mathrm{H}}=0
$$

Moreover, use of Eq. (41) into Eq. (40) allows to rewrite the equation in the equivalent form

$$
\left[\frac{\partial}{\partial s}+\boldsymbol{c}_{1} \cdot \frac{\partial}{\partial \boldsymbol{l}_{1}}-\Lambda\left(\boldsymbol{c}_{1}\right)\right] \delta \widetilde{F}_{1}\left(\boldsymbol{l}_{1}, \boldsymbol{c}_{1}, s\right)=\widetilde{S}\left(\boldsymbol{l}_{1}, \boldsymbol{c}_{1}, s\right)
$$

where

$$
\widetilde{S}\left(\boldsymbol{l}_{1}, \boldsymbol{c}_{1}, s\right) \equiv \int d \boldsymbol{c}_{2} \bar{T}_{0}\left(\boldsymbol{c}_{1}, \boldsymbol{c}_{2}\right) \widetilde{\Phi}_{2}^{B}\left(\boldsymbol{c}_{1}, \boldsymbol{c}_{2}, \boldsymbol{l}_{1}, s\right)
$$

and the operator $\Lambda\left(\boldsymbol{c}_{1}\right)$ was defined in Eq. (28). Equation (43) can be interpreted as a fluctuating Boltzmann-Langevin equation for the one-particle distribution function [8, 16, 17], with the "noise term" being $\widetilde{S}\left(\boldsymbol{l}_{1}, \boldsymbol{c}_{1}, s\right)$. Of course, this does add any new physical insight by itself in the understanding of the starting equation (34). The relevance and usefulness of this representation will depend on the properties of the noise term. A first one follows directly from Eq. (42), that is equivalent to

$$
\left\langle\widetilde{S}\left(\boldsymbol{l}_{1}, \boldsymbol{c}_{1}, s\right)\right\rangle_{\mathrm{H}}=0
$$

i.e. the noise has zero average. In the following, other properties of $\widetilde{S}$ will be derived by requiring consistency with the results derived in the previous section. Multiplication of Eq. (43) by $\delta \widetilde{F}_{1}\left(\boldsymbol{l}_{1}^{\prime}, \boldsymbol{c}_{1}^{\prime}, s^{\prime}\right)$ with $s^{\prime}<s$, followed by averaging gives

$$
\left[\frac{\partial}{\partial s}+\boldsymbol{c}_{1} \cdot \frac{\partial}{\partial \boldsymbol{l}}-\Lambda\left(\boldsymbol{c}_{1}\right)\right]\left\langle\delta \widetilde{F}_{1}\left(\boldsymbol{l}_{1}, \boldsymbol{c}_{1}, s\right) \delta \widetilde{F}_{1}\left(\boldsymbol{l}_{1}^{\prime}, \boldsymbol{c}_{1}^{\prime}, s^{\prime}\right)\right\rangle_{\mathrm{H}}=\left\langle\widetilde{S}\left(\boldsymbol{l}_{1}, \boldsymbol{c}_{1}, s\right) \delta \widetilde{F}_{1}\left(\boldsymbol{l}_{1}^{\prime}, \boldsymbol{c}_{1}^{\prime}, s^{\prime}\right)\right\rangle_{\mathrm{H}}
$$


From the definition of $\delta \widetilde{F}_{1}\left(\boldsymbol{l}_{1}, \boldsymbol{c}_{1}, s\right)$ it is easily verified that

$$
\left\langle\delta \widetilde{F}_{1}\left(\boldsymbol{l}_{1}, \boldsymbol{c}_{1}, s\right) \delta \widetilde{F}_{1}\left(\boldsymbol{l}_{1}^{\prime}, \boldsymbol{c}_{1}^{\prime}, s^{\prime}\right)\right\rangle_{\mathrm{H}}=n^{-1} \lambda^{-d} \widetilde{h}\left(\boldsymbol{l}_{1}-\boldsymbol{l}_{1}^{\prime}, \boldsymbol{c}_{1}, s-s^{\prime} ; \boldsymbol{c}_{1}^{\prime}\right),
$$

where $\widetilde{h}$ is defined in Eq. (29). Therefore, consistency of Eqs. (46) and (30) implies that

$$
\left\langle\widetilde{S}\left(\boldsymbol{l}_{1}, \boldsymbol{c}_{1}, s\right) \delta \widetilde{F}_{1}\left(\boldsymbol{l}_{1}^{\prime}, \boldsymbol{c}_{1}^{\prime}, s^{\prime}\right)\right\rangle_{\mathrm{H}}=0
$$

for $s>s^{\prime}$. Since, by hypothesis, the parameters of the system are such that the HCS is stable, the long time solution of Eq. (43) is

$$
\delta \widetilde{F}_{1}\left(\boldsymbol{l}_{1}, \boldsymbol{c}_{1}, s\right)=\int_{-\infty}^{s} d \tau e^{(s-\tau) L\left(\boldsymbol{l}_{1}, \boldsymbol{c}_{1}\right)} \widetilde{S}\left(\boldsymbol{l}_{1}, \boldsymbol{c}_{1}, \tau\right)
$$

where the linear operator

$$
L\left(\boldsymbol{l}_{1}, \boldsymbol{c}_{1}\right) \equiv \Lambda\left(\boldsymbol{c}_{1}\right)-\boldsymbol{c}_{1} \cdot \frac{\partial}{\partial \boldsymbol{l}_{1}}
$$

has been introduced. Using Eq. (49), it is obtained

$$
\begin{gathered}
{\left[L\left(\boldsymbol{l}_{1}, \boldsymbol{c}_{1}\right)+L\left(\boldsymbol{l}_{1}^{\prime}, \boldsymbol{c}_{1}^{\prime}\right)\right]\left\langle\delta \widetilde{F}_{1}\left(\boldsymbol{l}_{1}, \boldsymbol{c}_{1}, s\right) \delta \widetilde{F}_{1}\left(\boldsymbol{l}_{1}^{\prime}, \boldsymbol{c}_{1}^{\prime}, s^{\prime}\right)\right\rangle_{\mathrm{H}}} \\
=-\int_{-\infty}^{s} d \tau e^{(s-\tau) L\left(\boldsymbol{l}_{1}, \boldsymbol{c}_{1}\right)}\left\langle\widetilde{S}\left(\boldsymbol{l}_{1}, \boldsymbol{c}_{1}, \tau\right) \widetilde{S}\left(\boldsymbol{l}_{1}^{\prime}, \boldsymbol{c}_{1}^{\prime}, s\right)\right\rangle_{\mathrm{H}} \\
\quad-\int_{-\infty}^{s} d \tau e^{(s-\tau) L\left(\boldsymbol{l}_{1}^{\prime}, \boldsymbol{c}_{1}^{\prime}\right)}\left\langle\widetilde{S}\left(\boldsymbol{l}_{1}, \boldsymbol{c}_{1}, s\right) \widetilde{S}\left(\boldsymbol{l}_{1}^{\prime}, \boldsymbol{c}_{1}^{\prime}, \tau\right)\right\rangle_{\mathrm{H}} .
\end{gathered}
$$

This equation must be compared with Eq. (32), having in mind Eq. (47). The time independence of the right hand side of Eq. (32) prompts to introduce the hypothesis that the noise term $\widetilde{S}$ is Markovian, and write

$$
\left\langle\widetilde{S}\left(\boldsymbol{l}_{1}, \boldsymbol{c}_{1}, s\right) \widetilde{S}\left(\boldsymbol{l}_{1}^{\prime}, \boldsymbol{c}_{1}^{\prime}, s^{\prime}\right)\right\rangle_{\mathrm{H}}=H\left(\boldsymbol{c}_{1}, \boldsymbol{c}_{1}^{\prime}\right) \delta\left(\boldsymbol{l}_{1}-\boldsymbol{l}_{1}^{\prime}\right) \delta\left(s-s^{\prime}\right)
$$

On introduction of this into Eq. (51) and comparison with Eq. (32), it follows that

$$
H\left(\boldsymbol{c}_{1}, \boldsymbol{c}_{1}^{\prime}\right)=n^{-1} \lambda^{-d} \widetilde{\Gamma}\left(\boldsymbol{c}_{1}, \boldsymbol{c}_{1}^{\prime}\right)
$$

with $\widetilde{\Gamma}$ defined in Eq. (333).

The properties given by Eqs. (45), (48), and (52) guarantee that the description provided by the Langevin equation (43) leads to the same expressions for the two-particle, one-time and two-time correlation functions as the formulation in terms of reduced distributions functions reviewed in Sec. II. 


\section{FLUCTUATING HYDRODYNAMIC FIELDS AND BALANCE EQUATIONS}

The fluctuating number of particles density, $N(\boldsymbol{r}, t)$, momentum density, $\boldsymbol{G}(\boldsymbol{r}, t)$, and energy density, $E(\boldsymbol{r}, t)$, are defined in terms of the microscopic phase space density as

$$
\begin{gathered}
N(\boldsymbol{r}, t)=\int d \boldsymbol{v} F_{1}(x, t), \\
\boldsymbol{G}(\boldsymbol{r}, t)=\int d \boldsymbol{v} m \boldsymbol{v} F_{1}(x, t), \\
E(\boldsymbol{r}, t)=\int d \boldsymbol{v} \frac{m v^{2}}{2} F_{1}(x, t) .
\end{gathered}
$$

Dimensionless deviations from their averages values in the HCS are given by

$$
\begin{gathered}
\delta \rho(\boldsymbol{l}, s) \equiv \frac{\delta N(\boldsymbol{r}, t)}{n}=\int d \boldsymbol{c} \delta \widetilde{F}_{1}(\boldsymbol{l}, \boldsymbol{c}, s), \\
\delta \boldsymbol{\omega}(\boldsymbol{l}, s) \equiv \frac{\delta \boldsymbol{G}(\boldsymbol{r}, t)}{m n v_{0}(t)}=\int d \boldsymbol{c} \boldsymbol{c} \delta \widetilde{F}_{1}(\boldsymbol{l}, \boldsymbol{c}, s), \\
\delta \epsilon(\boldsymbol{l}, s) \equiv \frac{2 \delta E(\boldsymbol{r}, t)}{d n T(t)}=\frac{2}{d} \int d \boldsymbol{c} c^{2} \delta \widetilde{F}_{1}(\boldsymbol{l}, \boldsymbol{c}, s) .
\end{gathered}
$$

The quantity $\delta \boldsymbol{\omega}(\boldsymbol{l}, s)$ is the dimensionless velocity field. Balance equations for these fluctuating fields follow by taking velocity moments in the Langevin-Boltzmann equation (43) and using the properties of the noise $\widetilde{S}$. Some details of the calculations are given in appendix A. The resulting equations read

$$
\begin{gathered}
\frac{\partial}{\partial s} \delta \rho(\boldsymbol{l}, s)+\frac{\partial}{\partial \boldsymbol{l}} \cdot \delta \boldsymbol{\omega}(\boldsymbol{l}, s)=0 \\
\left(\frac{\partial}{\partial s}-\frac{\zeta_{0}}{2}\right) \delta \boldsymbol{\omega}(\boldsymbol{l}, s)+\frac{\partial}{\partial \boldsymbol{l}} \cdot \delta \Pi(\boldsymbol{l}, s)=0 \\
\left(\frac{\partial}{\partial s}-\zeta_{0}\right) \delta \epsilon(\boldsymbol{l}, s)+\frac{d+2}{d} \frac{\partial}{\partial \boldsymbol{l}} \cdot \delta \boldsymbol{\omega}(\boldsymbol{l}, s)+\delta \zeta_{0}(\boldsymbol{l}, s)+\frac{2}{d} \frac{\partial}{\partial \boldsymbol{l}} \cdot \delta \boldsymbol{\phi}(\boldsymbol{l}, s)=\widetilde{S}_{\epsilon}(\boldsymbol{l}, s) .
\end{gathered}
$$

In the above equations, $\delta \Pi(\boldsymbol{l}, s)$ and $\delta \boldsymbol{\phi}(\boldsymbol{l}, s)$ are the fluctuating pressure tensor and heat flux, respectively. Their definitions in terms of the fluctuating one-particle distribution function are

$$
\begin{gathered}
\delta \Pi(\boldsymbol{l}, s)=\frac{\delta \epsilon(\boldsymbol{l}, s)}{2} \mathrm{I}+\int d \boldsymbol{c} \Delta(\boldsymbol{c}) \delta \widetilde{F}_{1}(\boldsymbol{l}, \boldsymbol{c}, s), \\
\delta \boldsymbol{\phi}(\boldsymbol{l}, s)=\int d \boldsymbol{c} \boldsymbol{\Sigma}(\boldsymbol{c}) \delta \widetilde{F}_{1}(\boldsymbol{l}, \boldsymbol{c}, s)
\end{gathered}
$$


where I is the unit tensor of dimension $d$, and

$$
\begin{gathered}
\Delta(\boldsymbol{c}) \equiv \boldsymbol{c} \boldsymbol{c}-\frac{c^{2}}{d} \mathrm{I}, \\
\Sigma(\boldsymbol{c}) \equiv\left(c^{2}-\frac{d^{2}+2}{2}\right) \boldsymbol{c} .
\end{gathered}
$$

The term $\delta \zeta_{0}(\boldsymbol{l}, s)$ represents the fluctuations of the cooling rate about its average value in the HCS. Its formal expressions is

$$
\delta \zeta_{0}(\boldsymbol{l}, s)=\frac{\left(1-\alpha^{2}\right) \pi^{\frac{d-1}{2}}}{\Gamma\left(\frac{d+3}{2}\right) d} \int d \boldsymbol{c}_{1} \int d \boldsymbol{c}_{2} c_{12}^{3} \chi\left(c_{1}\right) \delta \widetilde{F}_{1}\left(\boldsymbol{l}, \boldsymbol{c}_{2}, s\right) .
$$

Finally, $\widetilde{S}_{\epsilon}(\boldsymbol{l}, s)$ is a fluctuating force term having the properties

$$
\left\langle\widetilde{S}_{\epsilon}(\boldsymbol{l}, s)\right\rangle_{\mathrm{H}}=0
$$

and

$$
\begin{aligned}
\left\langle\widetilde{S}_{\epsilon}(\boldsymbol{l}, s) \widetilde{S}_{\epsilon}\left(\boldsymbol{l}^{\prime}, s^{\prime}\right)\right\rangle_{\mathrm{H}}= & n^{-1} \lambda^{-d} \delta\left(s-s^{\prime}\right) \delta\left(\boldsymbol{l}-\boldsymbol{l}^{\prime}\right)\left[\int d \boldsymbol{c}_{1} \int d \boldsymbol{c}_{2} \chi\left(c_{1}\right) \chi\left(c_{2}\right) \Phi\left(\boldsymbol{c}_{1}, \boldsymbol{c}_{2}\right)\right. \\
& \left.-\frac{8}{d^{2}} \zeta_{0} \int d \boldsymbol{c} c^{4} \chi(c)\right],
\end{aligned}
$$

with $\Phi\left(\boldsymbol{c}_{1}, \boldsymbol{c}_{2}\right)$ given by Eq. (A16). This noise term is intrinsic to the inelasticity of collisions and has no analogue in normal fluids. Of course, in the elastic limit $\alpha \rightarrow 1, \chi(c)$ becomes

a Gaussian and the fluctuating force $\widetilde{S}_{\epsilon}$ is seen to vanish in agreement with the well known results for hydrodynamic fluctuations in molecular fluids [18]. The other main differences between Eq. (62) and the one for molecular gases is the presence of the two terms involving the cooling rate, $\zeta_{0}$, and its fluctuations, $\delta \zeta_{0}$. The presence of these terms is directly associated with existence of the cooling term in the macroscopic equation for the average energy $[3,30,31]$.

\section{LANGEVIN EQUATION FOR THE VELOCITY FIELD}

Equations (60)-(62) do not provide a closed description of the hydrodynamic fluctuations of a granular gas in the HCS until $\delta \Pi, \delta \phi$, and $\delta \zeta_{0}$ are expressed in terms of the fluctuating hydrodynamic fields. This turns out to be not a simple task, and attention will be restricted in the following to the equation of the velocity field $\delta \boldsymbol{\omega}(\boldsymbol{l}, s)$, Eq. (61). 
Given two functions $f(\boldsymbol{c})$ and $g(\boldsymbol{c})$, their scalar product is defined as

$$
\langle f \mid g\rangle \equiv \int d \boldsymbol{c} \chi^{-1}(c) f^{+}(\boldsymbol{c}) g(\boldsymbol{c})
$$

where $f^{+}(\boldsymbol{c})$ is the complex conjugate of $f(\boldsymbol{c})$. Next, a projection operator $\mathcal{P}$ is introduced by

$$
\mathcal{P} f(\boldsymbol{c}) \equiv \sum_{\beta=1}^{d+2} \xi_{\beta}(\boldsymbol{c})\left\langle\bar{\xi}_{\beta} \mid f\right\rangle .
$$

Here, the functions $\xi_{\beta}(\boldsymbol{c}), \beta=1, \ldots, d+2$ are the eigenfunctions of the linear Boltzmann operator $\Lambda(\boldsymbol{c})$ defined in Eq. (28), corresponding to the hydrodynamic part of its spectrum. Therefore, they are solutions of the equation

$$
\Lambda(\boldsymbol{c}) \xi_{\beta}(\boldsymbol{c})=\lambda_{\beta} \xi_{\beta}(\boldsymbol{c})
$$

Their expressions are [22, 29]

$$
\xi_{1}(\boldsymbol{c})=\chi(c)+\frac{\partial}{\partial \boldsymbol{c}} \cdot[\boldsymbol{c} \chi(c)], \quad \boldsymbol{\xi}_{2}(\boldsymbol{c})=-\frac{\partial \chi(c)}{\partial \boldsymbol{c}}, \quad \xi_{3}(\boldsymbol{c})=-\frac{\partial}{\partial \boldsymbol{c}} \cdot[\boldsymbol{c} \chi(c)] .
$$

The associated eigenvalues are found to be

$$
\lambda_{1}=0, \quad \lambda_{2}=\frac{\zeta_{0}}{2}, \quad \lambda_{3}=-\frac{\zeta_{0}}{2}
$$

the eigenvalue $\lambda_{2}$ being $d$-fold degenerated. Finally the functions $\bar{\xi}_{\beta}(\boldsymbol{c})$ are

$$
\bar{\xi}_{1}(\boldsymbol{c})=\chi(c), \quad \overline{\boldsymbol{\xi}}_{2}(\boldsymbol{c})=\boldsymbol{c} \chi(c), \quad \bar{\xi}_{3}(\boldsymbol{c})=\left(\frac{c^{2}}{d}+\frac{1}{2}\right) \chi(c)
$$

The sets of functions $\left\{\xi_{\beta}(\boldsymbol{c})\right\}$ and $\left\{\bar{\xi}_{\beta}(\boldsymbol{c})\right\}$ are seen to have the biorthogonality property

$$
\left\langle\bar{\xi}_{\beta} \mid \xi_{\beta^{\prime}}\right\rangle=\delta_{\beta, \beta^{\prime}}
$$

$\beta, \beta^{\prime}=1,2, \ldots, d+2$. This guarantees that $\mathcal{P}$ as defined by Eq. (71) is really a projector operator, i.e. it verifies $\mathcal{P}^{2}=\mathcal{P}$. It projects any function of $\boldsymbol{c}$ onto the subspace spanned by the hydrodynamic eigenfunctions of $\Lambda$.

In the following, it will be more convenient to work in the Fourier representation. The Fourier transform of $\delta \widetilde{F}_{1}(\boldsymbol{l}, \boldsymbol{c}, s)$ is

$$
\delta \widetilde{F}_{1}(\boldsymbol{k}, \boldsymbol{c}, s)=\int d \boldsymbol{l} e^{-i \boldsymbol{k} \cdot \boldsymbol{l}} \delta \widetilde{F}_{1}(\boldsymbol{l}, \boldsymbol{c}, s) .
$$


By means of $\mathcal{P}, \delta \widetilde{F}_{1}(\boldsymbol{k}, \boldsymbol{c}, s)$ can be decomposed into its hydrodynamic and nonhydrodynamic parts,

$$
\delta \widetilde{F}_{1}(\boldsymbol{k}, \boldsymbol{c}, s)=\mathcal{P} \delta \widetilde{F}_{1}(\boldsymbol{k}, \boldsymbol{c}, s)+\mathcal{P}_{\perp} \delta \widetilde{F}_{1}(\boldsymbol{k}, \boldsymbol{c}, s)
$$

where $\mathcal{P}_{\perp} \equiv 1-\mathcal{P}$. The Fourier representation of the balance equation for the velocity fluctuations, Eq. (61), is

$$
\begin{gathered}
\left(\frac{\partial}{\partial s}-\frac{\zeta_{0}}{2}\right) \delta \boldsymbol{\omega}(\boldsymbol{k}, s)+i \boldsymbol{k} \cdot \delta \Pi(\boldsymbol{k}, s)=0, \\
\delta \Pi(\boldsymbol{k}, s)=\frac{\delta \epsilon(\boldsymbol{k}, s)}{2} \mathrm{I}+\int d \boldsymbol{c} \Delta(\boldsymbol{c}) \delta \widetilde{F}_{1}(\boldsymbol{k}, \boldsymbol{c}, s),
\end{gathered}
$$

where $\Delta(\boldsymbol{c})$ is defined in Eq. (65). Getting an explicit expression for $\delta \Pi(\boldsymbol{k}, s)$ in terms of the fluctuating hydrodynamic fields is the next issue to be addressed. By direct evaluation, it is easily verified that

$$
\int d \boldsymbol{c} \Delta(\boldsymbol{c}) \xi_{\beta}(\boldsymbol{c})=0
$$

$\beta=1, \ldots, d+2$. Hence Eq. (80) is equivalent to

$$
\delta \Pi(\boldsymbol{k}, s)=\frac{\delta \epsilon(\boldsymbol{k}, s)}{2} \mathrm{I}+\int d \boldsymbol{c} \Delta(\boldsymbol{c}) \mathcal{P}_{\perp} \delta \widetilde{F}_{1}(\boldsymbol{k}, \boldsymbol{c}, s) .
$$

To compute $\mathcal{P}_{\perp} \widetilde{F}_{1}(\boldsymbol{k}, \boldsymbol{c}, s)$, the operator $\mathcal{P}_{\perp}$ is applied to both sides of the Fourier transform of the Boltzmann-Langevin equation (43),

$$
\left\{\frac{\partial}{\partial s}-\mathcal{P}_{\perp}[\Lambda(\boldsymbol{c})-i \boldsymbol{k} \cdot \boldsymbol{c}]\right\} \mathcal{P}_{\perp} \delta \widetilde{F}_{1}(\boldsymbol{k}, \boldsymbol{c}, s)=-\mathcal{P}_{\perp} i \boldsymbol{k} \cdot \boldsymbol{c} \mathcal{P} \delta \widetilde{F}_{1}(\boldsymbol{k}, \boldsymbol{c}, s)+\mathcal{P}_{\perp} \widetilde{S}(\boldsymbol{k}, \boldsymbol{c}, s)
$$

where use has been made of the property $\mathcal{P}_{\perp} \Lambda \mathcal{P}=0$, that is a consequence of the fact that $\mathcal{P}$ projects over a subspace generated by eigenfunctions of $\Lambda$. The solution of the above equation can be formally written as

$$
\begin{aligned}
\mathcal{P}_{\perp} \delta \widetilde{F}_{1}(\boldsymbol{k}, \boldsymbol{c}, s)= & \mathcal{U}(\boldsymbol{k}, \boldsymbol{c}, s) \mathcal{P}_{\perp} \delta \widetilde{F}_{1}(\boldsymbol{k}, \boldsymbol{c}, 0)+\int_{0}^{s} d s^{\prime} \mathcal{U}\left(\boldsymbol{k}, \boldsymbol{c}, s^{\prime}\right) \mathcal{P}_{\perp}\left[-i \boldsymbol{k} \cdot \boldsymbol{c} \mathcal{P} \delta \widetilde{F}_{1}\left(\boldsymbol{k}, \boldsymbol{c}, s-s^{\prime}\right)\right. \\
& \left.+\widetilde{S}\left(\boldsymbol{k}, \boldsymbol{c}, s-s^{\prime}\right)\right]
\end{aligned}
$$

with

$$
\begin{gathered}
\mathcal{U}(\boldsymbol{k}, \boldsymbol{c}, s) \equiv \exp \left[s \mathcal{P}_{\perp} L(\boldsymbol{k}, c) \mathcal{P}_{\perp}\right] \\
L(\boldsymbol{k}, \boldsymbol{c}) \equiv \Lambda(\boldsymbol{c})-i \boldsymbol{k} \cdot \boldsymbol{c}
\end{gathered}
$$


Taking again into account that the HCS is assumed to be stable for the system considered, the first term on the right hand side of Eq. (84) can be neglected for large enough times $s$. Moreover, to derive hydrodynamic equations valid to Navier-Stokes order, only the first order in $k$ of the pressure tensor is needed. To this order,

$$
\begin{aligned}
& \int_{0}^{s} d s^{\prime} \mathcal{U}\left(\boldsymbol{k}, \boldsymbol{c}, s^{\prime}\right) \mathcal{P}_{\perp}(-i \boldsymbol{k} \cdot \boldsymbol{c}) \mathcal{P} \delta \widetilde{F}_{1}\left(\boldsymbol{k}, \boldsymbol{c}, s-s^{\prime}\right) \\
\rightarrow & \int_{0}^{s} d s^{\prime} e^{s^{\prime} \mathcal{P}_{\perp} \Lambda(\boldsymbol{c}) \mathcal{P}_{\perp}} \mathcal{P}_{\perp}(-i \boldsymbol{k} \cdot \boldsymbol{c}) \mathcal{P} \delta \widetilde{F}_{1}\left(\boldsymbol{k}, \boldsymbol{c}, s-s^{\prime}\right) \\
= & \int_{0}^{s} d s^{\prime} \mathcal{P}_{\perp} e^{s^{\prime} \Lambda(\boldsymbol{c})}(-i \boldsymbol{k} \cdot \boldsymbol{c}) \mathcal{P} \delta \widetilde{F}_{1}\left(\boldsymbol{k}, \boldsymbol{c}, s-s^{\prime}\right) .
\end{aligned}
$$

Then, for large $s$ Eq. (84) reduces to

$$
\begin{aligned}
\mathcal{P}_{\perp} \delta \widetilde{F}_{1}(\boldsymbol{k}, \boldsymbol{c}, s)= & \int_{0}^{s} d s^{\prime} \mathcal{P}_{\perp} e^{s^{\prime} \Lambda(\boldsymbol{c})}(-i \boldsymbol{k} \cdot \boldsymbol{c}) \mathcal{P} \delta \widetilde{F}_{1}\left(\boldsymbol{k}, \boldsymbol{c}, s-s^{\prime}\right) \\
& +\int_{0}^{s} d s^{\prime} \mathcal{U}\left(\boldsymbol{k}, \boldsymbol{c}, s^{\prime}\right) \mathcal{P}_{\perp} \widetilde{S}\left(\boldsymbol{k}, \boldsymbol{c}, s-s^{\prime}\right)
\end{aligned}
$$

and substitution of this into Eq. (82) yields

$$
\delta \Pi(\boldsymbol{k}, s)=\frac{\delta \epsilon(\boldsymbol{k}, s)}{2} \mathrm{I}+\delta_{1} \Pi(\boldsymbol{k}, s)+\mathrm{R}(\boldsymbol{k}, s),
$$

where

$$
\delta_{1} \Pi(\boldsymbol{k}, s)=\int_{0}^{s} d s^{\prime} \int d \boldsymbol{c} \Delta(\boldsymbol{c}) e^{s^{\prime} \Lambda(\boldsymbol{c})}(-i \boldsymbol{k} \cdot \boldsymbol{c}) \mathcal{P} \delta \widetilde{F}_{1}\left(\boldsymbol{k}, \boldsymbol{c}, s-s^{\prime}\right)
$$

and

$$
\mathrm{R}(\boldsymbol{k}, s)=\int_{0}^{s} d s^{\prime} \int d \boldsymbol{c} \Delta(\boldsymbol{c}) \mathcal{U}\left(\boldsymbol{k}, \boldsymbol{c}, s^{\prime}\right) \mathcal{P}_{\perp} \widetilde{S}\left(\boldsymbol{k}, \boldsymbol{c}, s-s^{\prime}\right) .
$$

Upon writing Eq. (90), Eq. (81) has been employed to remove the operator $\mathcal{P}_{\perp}$ appearing in the first term on the right hand side of Eq. (88). Because of the isotropy of the operator $\Lambda(\boldsymbol{c})$, only the projection onto $\boldsymbol{\xi}_{2}(\boldsymbol{c})$ gives a non-vanishing contribution to the above expression for $\delta_{1} \Pi(\boldsymbol{k}, s)$, that can be simplified to

$$
\begin{aligned}
\delta_{1} \Pi(\boldsymbol{k}, s) & =\int_{0}^{s} d s^{\prime} \int d \boldsymbol{c} \Delta(\boldsymbol{c}) e^{s^{\prime} \Lambda(\boldsymbol{c})}(-i \boldsymbol{k} \cdot \boldsymbol{c}) \boldsymbol{\xi}_{2}(\boldsymbol{c}) \cdot\left\langle\overline{\boldsymbol{\xi}}_{2}(\boldsymbol{c}) \mid \delta \widetilde{F}_{1}\left(\boldsymbol{k}, \boldsymbol{c}, s-s^{\prime}\right)\right\rangle \\
& =\int_{0}^{s} d s^{\prime} \int d \boldsymbol{c} \Delta(\boldsymbol{c}) e^{s^{\prime} \Lambda(\boldsymbol{c})}(-i \boldsymbol{k} \cdot \boldsymbol{c}) \boldsymbol{\xi}_{2}(\boldsymbol{c}) \cdot \delta \boldsymbol{\omega}\left(\boldsymbol{k}, s-s^{\prime}\right) \\
& \simeq \int_{0}^{s} d s^{\prime} \int d \boldsymbol{c} \Delta(\boldsymbol{c}) e^{s^{\prime} \Lambda(\boldsymbol{c})}(-i \boldsymbol{k} \cdot \boldsymbol{c}) e^{-s^{\prime} \zeta_{0} / 2} \boldsymbol{\xi}_{2}(\boldsymbol{c}) \cdot \delta \boldsymbol{\omega}(\boldsymbol{k}, s) .
\end{aligned}
$$


In the previous transformations, the definition in Eq. (58) has been used, and it has been taken into account that to lowest order in $k$,

$$
\delta \omega\left(\boldsymbol{k}, s-s^{\prime}\right)=e^{-s^{\prime} \zeta_{0} / 2} \delta \omega(\boldsymbol{k}, s)
$$

according to the balance equation for the fluctuating velocity field, Eq. (61). Using again the symmetry of $\boldsymbol{\Lambda}(\boldsymbol{c})$, it is obtained:

$$
\delta_{1} \Pi_{i j}(\boldsymbol{k}, s)=-i \widetilde{\eta}(s)\left[k_{i} \delta \omega_{j}(\boldsymbol{k}, s)+k_{j} \delta \omega_{i}(\boldsymbol{k}, s)-\frac{2}{d} \delta_{i j} \boldsymbol{k} \cdot \delta \widetilde{\boldsymbol{\omega}}(\boldsymbol{k}, s)\right] .
$$

This is the same as the Navier-Stokes expression for the pressure tensor with the only difference that the average macroscopic velocity field is substituted by the fluctuating one. It involves the (time-dependent) dimensionless shear viscosity $\widetilde{\eta}(s)$ defined by

$$
\widetilde{\eta}(s)=\frac{1}{d^{2}+d-2} \sum_{i} \sum_{j} \int_{0}^{s} d s^{\prime} \int d \mathbf{c} \Delta_{i j}(\boldsymbol{c}) e^{s^{\prime}\left(\Lambda-\frac{\zeta_{0}}{2}\right)} \xi_{2, i}(\boldsymbol{c}) c_{j} .
$$

This expression is equivalent to the one obtained from the nonlinear Boltzmann equation for inelastic hard spheres or disks by the Chapman-Enskog method [30, 31] and also to the Green-Kubo formulas derived in ref. [32]. Let us remark that the results obtained here apply in the limit of large $s$. It is in this limit when hydrodynamics in the usual sense is expected to apply. If this is true, the shear viscosity in Eq. (95) will become independent of $s$. Although there is no a mathematical proof of this "ageing to hydrodynamics" for granular gases up to now, numerical evaluation of the right hand side of Eq. (95) by using the direct Monte Carlo simulation method has shown the existence of such a limit value [33]. Moreover, the simulation results for the shear viscosity $\widetilde{\eta}$ are in good agreement with the expression obtained by evaluating the Chapman-Enskog result in the first Sonine approximation [30, 31],

$$
\begin{gathered}
\widetilde{\eta}(\alpha)=\left[8 \widetilde{\nu}(\alpha)-\zeta_{0}(\alpha)\right]^{-1}, \\
\widetilde{\nu}(\alpha)=\frac{\pi^{\frac{d-1}{2}}}{2 \sqrt{2} d(d+2) \Gamma(d / 2)}(3-3 \alpha+2 d)(1+\alpha)\left[1-\frac{a_{2}(\alpha)}{32}\right] .
\end{gathered}
$$

When Eq. (94) is substituted into Eq. (89) and the resulting expression is used into Eq. (79), a Langevin-like equation is obtained for the velocity field,

$$
\left(\frac{\partial}{\partial s}-\frac{\zeta_{0}}{2}\right) \delta \boldsymbol{\omega}(\boldsymbol{k}, s)+\frac{i}{2} \delta \epsilon(\boldsymbol{k}, s) \boldsymbol{k}+\widetilde{\eta}\left[k^{2} \delta \boldsymbol{\omega}(\boldsymbol{k}, s)+\frac{d-2}{d} \boldsymbol{k} \cdot \delta \boldsymbol{\omega}(\boldsymbol{k}, s) \boldsymbol{k}\right]=\boldsymbol{W}(\boldsymbol{k}, s),
$$


with the noise term given by

$$
\boldsymbol{W}(\boldsymbol{k}, s) \equiv-i \boldsymbol{k} \cdot \mathrm{R}(\boldsymbol{k}, s),
$$

where the term $\mathrm{R}(\boldsymbol{k}, s)$ is defined in Eq. (91). It follows from Eq. (45) that

$$
\langle\boldsymbol{W}(\boldsymbol{k}, s)\rangle_{\mathrm{H}}=0
$$

A formal expression for the correlation function of $\boldsymbol{W}$ is obtained directly by using Eq. (52). Its conversion into an explicit one, valid to Navier-Stokes order and, therefore, consistent with the left hand side of Eq. (98), will be carried out in the next section for the particular case of the transverse component of the velocity field.

\section{THE NOISE TERM IN THE EQUATION FOR THE TRANSVERSE VELOC- ITY FIELD}

The transverse part of the fluctuating velocity field, $\delta \boldsymbol{\omega}_{\perp}(\boldsymbol{k}, s)$, is defined by

$$
\delta \boldsymbol{\omega}_{\perp}(\boldsymbol{k}, s) \equiv \delta \boldsymbol{\omega}(\boldsymbol{k}, s)-\delta \boldsymbol{\omega}(\boldsymbol{k}, s) \cdot \frac{\boldsymbol{k}}{k^{2}} \boldsymbol{k} .
$$

Its evolution equation can be written down directly from Eq. (98),

$$
\left(\frac{\partial}{\partial s}-\frac{\zeta_{0}}{2}+\widetilde{\eta} k^{2}\right) \delta \boldsymbol{\omega}_{\perp}(\boldsymbol{k}, s)=\boldsymbol{W}_{\perp}(\boldsymbol{k}, s)
$$

where

$$
\boldsymbol{W}_{\perp}(\boldsymbol{k}, s)=-i \boldsymbol{k}\left(\mathrm{I}-\frac{\boldsymbol{k} \boldsymbol{k}}{k^{2}}\right): \mathrm{R}(\boldsymbol{k}, s) .
$$

Substitution of the expression of R given in Eq. (91) yields

$$
\boldsymbol{W}_{\perp}(\boldsymbol{k}, s)=-i \int_{0}^{s} d s^{\prime} \int d \boldsymbol{c} \boldsymbol{k} \cdot \boldsymbol{c}\left(\boldsymbol{c}-\frac{\boldsymbol{c} \cdot \boldsymbol{k}}{k^{2}} \boldsymbol{k}\right) \mathcal{U}\left(\boldsymbol{k}, \boldsymbol{c}, s^{\prime}\right) \mathcal{P}_{\perp} \widetilde{S}\left(\boldsymbol{k}, \boldsymbol{c}, s-s^{\prime}\right) .
$$

By using this expression, it is shown in appendix $\mathrm{B}$ that for two components, $W_{\perp, i}(\boldsymbol{k}, s)$ and $W_{\perp, j}(\boldsymbol{k}, s)$, of the noise of the transverse velocity field, to lowest order in $k$ it is

$$
\left\langle W_{\perp, i}(\boldsymbol{k}, s) W_{\perp, j}\left(\boldsymbol{k}^{\prime}, s^{\prime}\right)\right\rangle_{\mathrm{H}}=\delta_{i, j} \delta_{\boldsymbol{k},-\boldsymbol{k}^{\prime}} \frac{\widetilde{V}^{2}}{N} k^{2} G\left(\left|s-s^{\prime}\right|\right),
$$

for $s>s^{\prime} \gg 1$. Here $\widetilde{V}$ is the volume of the system in the length scale $l$, i.e. $\widetilde{V} \equiv N / n \lambda^{d}$, and

$$
G(|s|)=\int d \boldsymbol{c} \int d \boldsymbol{c}^{\prime} \Delta_{x y}(\boldsymbol{c}) \Delta_{x y}\left(\boldsymbol{c}^{\prime}\right) \widetilde{\psi}_{\mathrm{HCS}}\left(\boldsymbol{c}, s ; \boldsymbol{c}^{\prime}\right)
$$


with

$$
\widetilde{\psi}_{\mathrm{HCS}}\left(\boldsymbol{c}, s ; \boldsymbol{c}^{\prime}\right)=\int d \boldsymbol{l} \widetilde{h}\left(\boldsymbol{l}, \boldsymbol{c}, s ; \boldsymbol{c}^{\prime}\right)
$$

The distribution $\widetilde{h}\left(\boldsymbol{l}, \boldsymbol{c}, s ; \boldsymbol{c}^{\prime}\right)$ is defined in Eq. (29)). Then, by integration of Eq. (30) it follows that $\widetilde{\psi}_{\text {HCS }}\left(\boldsymbol{c}, s ; \boldsymbol{c}^{\prime}\right)$ obeys the equation

$$
\left[\frac{\partial}{\partial s}-\Lambda(\boldsymbol{c})\right] \widetilde{\psi}_{\mathrm{HCS}}\left(\boldsymbol{c}, s ; \boldsymbol{c}^{\prime}\right)=0
$$

valid for $s>0$. In principle, this equation must be solved with the initial condition $\widetilde{\psi}_{\mathrm{HCS}}\left(\boldsymbol{c} ; \boldsymbol{c}^{\prime}\right)$, given by

$$
\widetilde{\psi}_{\mathrm{HCS}}\left(\boldsymbol{c} ; \boldsymbol{c}^{\prime}\right)=\int d \boldsymbol{l} \widetilde{g}\left(\boldsymbol{l}, \boldsymbol{c}, \boldsymbol{c}^{\prime}\right)+\delta\left(\boldsymbol{c}-\boldsymbol{c}^{\prime}\right) \chi(c),
$$

obtained by integration of Eq. (31). Nevertheless, it has been shown in ref [33] by particle simulation that contributions from the correlations in the HCS of dilute granular gases are negligible, at least for not too strong inelasticity, $\alpha \gtrsim 0.5$. Therefore, the term involving $\widetilde{g}$ in Eq. (109) has been neglected in the results reported below.

The solution of Eq. (102) in the limit of large $s$ can be written as

$$
\delta \omega_{\perp, i}(\boldsymbol{k}, s)=\int_{-\infty}^{s} d s_{1} e^{\lambda_{\perp}(k)\left(s-s_{1}\right)} W_{\perp, i}(\boldsymbol{k}, s),
$$

where

$$
\lambda_{\perp}(k) \equiv \frac{\zeta_{0}}{2}-\widetilde{\eta} k^{2} .
$$

Next, using Eq. (105) it is obtained that

$$
\left\langle\delta \omega_{\perp, i}(\boldsymbol{k}, s) \delta \omega_{\perp, i}\left(\boldsymbol{k}^{\prime}, s\right)\right\rangle_{\mathrm{H}}=-\frac{\widetilde{V}^{2}}{2 N} k^{2} \delta_{\boldsymbol{k},-\boldsymbol{k}^{\prime}} \frac{\widetilde{\eta}^{\prime}}{\lambda_{\perp}(k)},
$$

with the coefficient $\widetilde{\eta}^{\prime}$ defined as

$$
\widetilde{\eta}^{\prime}=2 \int_{0}^{\infty} d s G(|s|) e^{\frac{\zeta_{0}}{2}}
$$

This coefficient can be computed in the first Sonine approximation. Some details of the calculations are given in appendix C. The result reads

$$
\widetilde{\eta}^{\prime}=\frac{1+a_{2}(\alpha)}{8 \widetilde{\nu}(\alpha)-3 \zeta_{0}(\alpha)}
$$

where $\widetilde{\nu}(\alpha)$ was defined in Eq. (97). 
The two-time self-correlation function of the transverse velocity field can also be computed from Eqs. (105) and (110). Again, details of the calculations are given in appendix Cl For $s-s^{\prime}>0$, it is obtained

$$
\left\langle\delta \omega_{\perp, i}(\boldsymbol{k}, s) \delta \omega_{\perp, j}\left(\boldsymbol{k}^{\prime}, s^{\prime}\right)\right\rangle_{H} \simeq-\frac{\widetilde{V}^{2}}{2 N} \delta_{i, j} \delta_{\boldsymbol{k},-\boldsymbol{k}^{\prime}} \frac{\left(\widetilde{\eta}^{\prime}+\widetilde{\eta}_{1}\right) k^{2}}{\lambda_{\perp}(k)} e^{\lambda_{\perp}(k)\left(s-s^{\prime}\right)},
$$

where

$$
\widetilde{\eta}_{1}=\int_{0}^{\infty} d s G(|s|)\left[e^{-\lambda_{\perp}(k) s}-e^{\lambda_{\perp}(k) s}\right]
$$

It is worth to stress that this result only holds after a transient interval $s-s^{\prime}$, and for this

reason it does not reduce to Eq. (112) for $s=s^{\prime}$. On the other hand, the coefficient $\widetilde{\eta}_{1}$ can be expected to be small, since the second factor in the integrand remains small for the decay of the first one.

To check the theory developed along the paper, Eqs. (115) and (112) has been used to measure the shear viscosity $\widetilde{\eta}$ and $\widetilde{\eta}^{\prime}$ by means of molecular dynamics simulation of dilute granular gases. The results turn out to be in qualitative agreement with the theory, in the sense that the scaled one-time correlation function is independent of the variable $s$, and the two time correlation function only depends on the difference $s-s^{\prime}$ and decays exponentially after a short transient period. More details of the simulation method employed and the analysis of the data is given in [34]. The comparison between the values of $\widetilde{\eta}$ and $\widetilde{\eta}^{\prime}$, obtained from the simulation results and the theoretical predictions given by Eqs. (96) and (114), respectively is shown in Fig. 1. Of course, all the simulation data have been obtained with low density systems in the HCS. It can be observed that the agreement is very good over a quite range of values of the restitution coefficient $\alpha$, then providing a very strong support for both the theory developed here and the specific algorithm used to compute the coefficients.

\section{SUMMARY AND DISCUSSION}

The primary objective here has been to investigate hydrodynamic fluctuations in the homogeneous cooling state of dilute granular gases, modeled as an ensemble of inelastic hard particles. From this point of view, the fluctuating balance equations (60)-(62), together with the fluctuating Boltzmann equation (43) provide a solid starting point. The remaining task is to construct explicit expressions for the fluctuating flux and the cooling rate in terms of the 


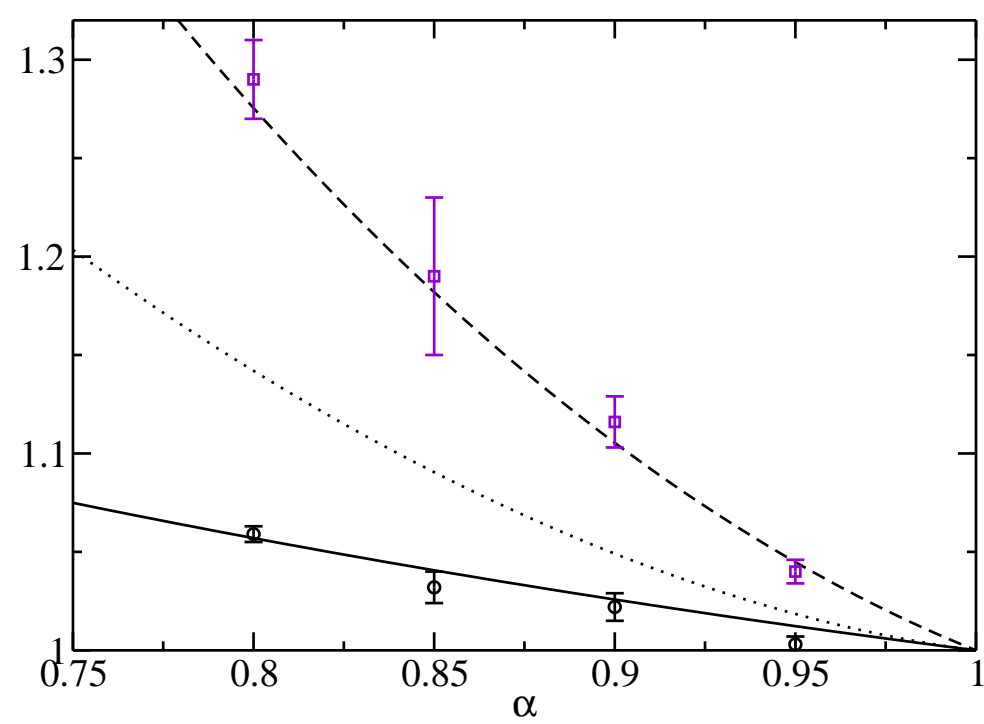

FIG. 1: (Color online) The shear viscosity $\widetilde{\eta}$ and the new coefficient $\widetilde{\eta}^{\prime}$ determining the transverse velocity fluctuations in granular gases in the HCS. The solid and dashed lines are the theoretical predictions for $\widetilde{\eta}$ and $\widetilde{\eta}^{\prime}$ given by Eqs. (96) and (114), respectively, normalized by the elastic value of the shear viscosity, $\widetilde{\eta}_{e}$. The circles $\left(\widetilde{\eta} / \widetilde{\eta}_{e}\right)$ and squares $\left(\widetilde{\eta}^{\prime} / \widetilde{\eta}_{e}\right)$ are molecular dynamics simulation results. The dotted line is the result obtained in the white noise approximation, Eq. (119).

(fluctuating) hydrodynamic fields by using, for instance, the Chapman-Enskog procedure. This part of the analysis turns out to be technically rather complex, and has been limited here to the particular case of the transverse flow field.

The structure of the fluctuating balance equations is similar to those for elastic, molecular systems with two main differences, both in the equation for the energy, Eq. (621). The equation contains a term, $\delta \zeta_{0}$, associated to the fluctuations of the cooling rate and also an intrinsic noise term $\widetilde{S}_{\epsilon}$. Both give contributions even to zeroth order in the gradients and therefore play a relevant role in describing the fluctuations of global properties of the system [13].

With regards to the fluctuating transverse velocity field, it has been found that it can be described by a Langevin equation, but exhibiting two crucial differences as compared with the elastic case. The form of the fluctuation dissipation relation changes both qualitatively and quantitatively. The second moment of the noise term is not determined by the shear viscosity. In addition, the noise is not white, i.e. it presents memory effects. Both aspects have been confirmed by the results obtained by molecular dynamics simulations. 
It could be wondered at what extension the memory effects mentioned above are relevant. Suppose the hypothesis of a white noise would have made and Eq. (105) were substituted by

$$
\left\langle W_{\perp, i}(\boldsymbol{k}, s) W_{\perp, j}\left(\boldsymbol{k}^{\prime}, s^{\prime}\right)\right\rangle_{\mathrm{H}}=\delta_{i, j} \delta_{\boldsymbol{k},-\boldsymbol{k}^{\prime}} \frac{\widetilde{V}^{2}}{N} k^{2} \widetilde{\eta}^{\prime \prime} \delta\left(s-s^{\prime}\right),
$$

with

$$
\widetilde{\eta}^{\prime \prime}=2 \int_{0}^{\infty} d s G(|s|) .
$$

By using the same method as outlined in appendix $[\mathbf{C}$ it is found that

$$
\widetilde{\eta}^{\prime \prime}=\frac{1+a_{2}(\alpha)}{8 \widetilde{\nu}(\alpha)-2 \zeta_{0}(\alpha)}
$$

This coefficient is also plotted in Fig. 1, and it is seen to clearly underestimate the amplitude of the second moment of the noise measured in the simulation. It is worth to stress that the violation of the elastic fluctuation-dissipation relations is already significant for values of the restitution coefficient $\alpha$ of the order of 0.95 .

\section{ACKNOWLEDGEMENTS}

This research was supported by the Ministerio de Educación y Ciencia (Spain) through Grant No. FIS2008-01339 (partially financed by FEDER funds). M.I.G.S. acknowledges financial support from Becas de la Fundación La Caixa and the French Government .

\section{APPENDIX A: DERIVATION OF THE FLUCTUATING HYDRODYNAMIC EQUATIONS}

In this appendix the derivation of Eqs. (60)facilitated by using that, for arbitrary functions $f\left(\boldsymbol{c}_{1}, \boldsymbol{c}_{2}\right)$ and $g\left(\boldsymbol{c}_{1}, \boldsymbol{c}_{2}\right)$, it is

$$
\begin{aligned}
& \int d \boldsymbol{c}_{1} \int d \boldsymbol{c}_{2} f\left(\boldsymbol{c}_{1}, \boldsymbol{c}_{2}\right) \bar{T}_{0}\left(\boldsymbol{c}_{1}, \boldsymbol{c}_{2}\right) g\left(\boldsymbol{c}_{1}, \boldsymbol{c}_{2}\right) \\
& =\int d \boldsymbol{c}_{1} \int d \boldsymbol{c}_{2} g\left(\boldsymbol{c}_{1}, \boldsymbol{c}_{2}\right) T_{0}\left(\boldsymbol{c}_{1}, \boldsymbol{c}_{2}\right) f\left(\boldsymbol{c}_{1}, \boldsymbol{c}_{2}\right),
\end{aligned}
$$

where

$$
T_{0}\left(\boldsymbol{c}_{1}, \boldsymbol{c}_{2}\right)=\int d \widehat{\boldsymbol{\sigma}} \Theta\left(\mathbf{c}_{12} \cdot \widehat{\boldsymbol{\sigma}}\right) \boldsymbol{c}_{12} \cdot \widehat{\boldsymbol{\sigma}}\left[b_{\boldsymbol{\sigma}}\left(\boldsymbol{c}_{1}, \boldsymbol{c}_{2}\right)-1\right]
$$


The operator $b_{\boldsymbol{\sigma}}$ is the inverse of $b_{\boldsymbol{\sigma}}^{-1}$ defined in Eq. (6). From Eqs. (57) and (43),

$\frac{\partial}{\partial s} \delta \rho(\boldsymbol{l}, s)=\int d \boldsymbol{c} \frac{\partial}{\partial s} \delta \widetilde{F}_{1}(\boldsymbol{l}, \boldsymbol{c}, s)=-\int d \boldsymbol{c} \boldsymbol{c} \cdot \frac{\partial}{\partial \boldsymbol{l}} \delta \widetilde{F}_{1}(\boldsymbol{l}, \boldsymbol{c}, s)+\int d \boldsymbol{c} \Lambda(\boldsymbol{c}) \delta \widetilde{F}_{1}(\boldsymbol{l}, \boldsymbol{c}, s)+\widetilde{S}_{\rho}(\boldsymbol{l}, s)$,

with

$$
\widetilde{S}_{\rho}(\boldsymbol{l}, s) \equiv \int d \boldsymbol{c} \widetilde{S}(\boldsymbol{l}, \boldsymbol{c}, s)
$$

By using the definition given in Eq. (28) and the property (A1) it is easy to see that

$$
\int d \boldsymbol{c} \Lambda(\boldsymbol{c}) \delta \widetilde{F}_{1}(\boldsymbol{l}, \boldsymbol{c}, s)=0
$$

Moreover,

$$
\begin{aligned}
\left\langle\widetilde{S}_{\rho}(\boldsymbol{l}, s) \widetilde{S}_{\rho}\left(\boldsymbol{l}^{\prime}, s^{\prime}\right)\right\rangle_{\mathrm{H}}= & \int d \boldsymbol{c}_{1} \int d \boldsymbol{c}_{2}\left\langle\widetilde{S}\left(\boldsymbol{l}, \boldsymbol{c}_{1}, s\right) \widetilde{S}\left(\boldsymbol{l}, \boldsymbol{c}_{2}, s\right)\right\rangle_{\mathrm{H}} \\
& =\delta\left(s-s^{\prime}\right) \delta\left(\boldsymbol{l}-\boldsymbol{l}^{\prime}\right) n^{-1} \lambda^{-d} \int d \boldsymbol{c}_{1} \int d \boldsymbol{c}_{2} \widetilde{\Gamma}\left(\boldsymbol{c}_{1}, \boldsymbol{c}_{2}\right),
\end{aligned}
$$

where Eqs. (52) and (53) have been employed. But, use again of Eq. (A1) yields

$$
\int d \boldsymbol{c}_{1} \int d \boldsymbol{c}_{2} \widetilde{\Gamma}\left(\boldsymbol{c}_{1}, \boldsymbol{c}_{2}\right)=0
$$

and consequently the second moment of $\widetilde{S}_{\rho}$ vanishes implying that the noise itself also identically vanishes. When all the above results are substituted into Eq. (A3) and the definition in Eq. (58) is taken into account, Eq. (60) follows directly.

The balance equation for the fluctuating velocity field is obtained in a similar way. Multiplication of Eq. (43) by $\boldsymbol{c}_{1}$ and integration over it gives

$$
\frac{\partial}{\partial s} \delta \boldsymbol{\omega}\left(\boldsymbol{l}_{1}, s\right)=-\frac{\partial}{\partial \boldsymbol{l}_{1}} \cdot \delta \Pi\left(\boldsymbol{l}_{1}, s\right)+\int d \boldsymbol{c}_{1} \boldsymbol{c}_{1} \Lambda\left(\boldsymbol{c}_{1}\right) \delta \widetilde{F}_{1}\left(\boldsymbol{l}_{1}, \boldsymbol{c}_{1}, s\right) \widetilde{\boldsymbol{S}}_{\omega}\left(\boldsymbol{l}_{1}, s\right),
$$

where $\Pi$ is defined in Eq. (63) and

$$
\widetilde{\boldsymbol{S}}_{\omega}\left(\boldsymbol{l}_{1}, s\right) \equiv \int d \boldsymbol{c}_{1} \boldsymbol{c}_{1} \widetilde{S}\left(\boldsymbol{l}_{1}, \boldsymbol{c}_{1}, s\right)
$$

A simple calculation, using Eq. (A1) and taking into account the momentum conservation in collisions leads to

$$
\int d \boldsymbol{c}_{1} \boldsymbol{c}_{1} \Lambda\left(\boldsymbol{c}_{1}\right) \delta \widetilde{F}_{1}\left(\boldsymbol{l}_{1}, \boldsymbol{c}_{1}, s\right)=\frac{\zeta_{0}}{2} \delta \boldsymbol{\omega}\left(\boldsymbol{l}_{1}, s\right)
$$


For the correlation of the noise $\widetilde{\boldsymbol{S}}_{\omega}$ by means of Eq. (152) it is found

$$
\begin{aligned}
\left\langle\widetilde{\boldsymbol{S}}_{\omega}\left(\boldsymbol{l}_{1}, s\right) \widetilde{\boldsymbol{S}}_{\omega}\left(\boldsymbol{l}_{1}^{\prime}, s\right)\right\rangle_{\mathrm{H}}= & -\delta\left(s-s^{\prime}\right) \delta\left(\boldsymbol{l}_{1}-\boldsymbol{l}_{1}^{\prime}\right) n^{-1} \lambda^{-d} \int d \boldsymbol{c}_{1} \int d \boldsymbol{c}_{1}^{\prime} \boldsymbol{c}_{1} \boldsymbol{c}_{1}^{\prime}\left\{\left[\Lambda\left(\boldsymbol{c}_{1}\right)+\Lambda\left(\boldsymbol{c}_{1}^{\prime}\right)\right]\right. \\
& \left.\times \delta\left(\boldsymbol{c}_{1}-\boldsymbol{c}_{1}^{\prime}\right) \chi\left(c_{1}\right) \bar{T}_{0}\left(\boldsymbol{c}_{1}, \boldsymbol{c}_{1}^{\prime}\right) \chi\left(c_{1}\right) \chi\left(c_{1}^{\prime}\right)\right\}
\end{aligned}
$$

The calculation of the first term on the right hand side of the above equation is straightforward giving

$$
\int d \boldsymbol{c}_{1} \int d \boldsymbol{c}_{1}^{\prime} \boldsymbol{c}_{1} \boldsymbol{c}_{1}^{\prime}\left[\Lambda\left(\boldsymbol{c}_{1}\right)+\Lambda\left(\boldsymbol{c}_{1}^{\prime}\right)\right] \delta\left(\boldsymbol{c}_{1}-\boldsymbol{c}_{1}^{\prime}\right) \chi\left(c_{1}\right)=\zeta_{0} \int d \boldsymbol{c}_{1} \boldsymbol{c}_{1} \boldsymbol{c}_{1} \chi\left(c_{1}\right)=\frac{\zeta_{0}}{2} \mathrm{I},
$$

where $\mathrm{I}$ is the unit tensor of dimension $d$. The evaluation of the last term on the right hand side of Eq. (A11) involves calculating several standard angular integrals. The result is

$$
\int d \boldsymbol{c}_{1} \int d \boldsymbol{c}_{1}^{\prime} \boldsymbol{c}_{1} \boldsymbol{c}_{1}^{\prime} \bar{T}_{0}\left(\boldsymbol{c}_{1}, \boldsymbol{c}_{1}^{\prime}\right) \chi\left(c_{1}\right) \chi\left(c_{1}^{\prime}\right)=\frac{\left(1-\alpha^{2}\right) \pi^{\frac{d-1}{2}}}{4 \Gamma\left(\frac{d+3}{2}\right) d} \mathbf{I} \int d \boldsymbol{c}_{1} \int d \boldsymbol{c}_{1}^{\prime} c_{11^{\prime}}^{3} \chi\left(c_{1}\right) \chi\left(c_{1}^{\prime}\right)=\frac{\zeta_{0}}{2} \mathrm{I} .
$$

Substitution of Eqs. (A12) and (A13) into Eq. (A11) shows that the second moment of the noise $\boldsymbol{S}_{\omega}$ vanishes implying that the noise itself also vanish identically. Then Eqs. (A8) and (A10) lead to Eq. (61).

Finally, multiplication of Eq. (43) by $2 c_{1}^{2} / d$ and integration over $\boldsymbol{c}_{1}$ yields

$$
\begin{aligned}
\frac{\partial}{\partial s} \delta \epsilon\left(\boldsymbol{l}_{1}, s\right)= & -\frac{2}{d} \frac{\partial}{\partial \boldsymbol{l}_{1}} \cdot \delta \phi\left(\boldsymbol{l}_{1}, s\right)+\frac{d+2}{d} \frac{\partial}{\partial \boldsymbol{l}_{1}} \cdot \delta \omega\left(\boldsymbol{l}_{1}, s\right) \\
& +\frac{2}{d} \int d \boldsymbol{c}_{1} c_{1}^{2} \Lambda\left(\boldsymbol{c}_{1}\right) \delta \widetilde{F}_{1}\left(\boldsymbol{l}_{1}, \boldsymbol{c}_{1}, s\right)+\widetilde{S}_{\epsilon}\left(\boldsymbol{l}_{1}, s\right),
\end{aligned}
$$

with the heat flux $\delta \phi$ defined by Eq. (64). Given that the kinetic energy is not conserved in collisions, the calculation of the second term on the right hand side of the above equation is more involved than in previous cases, although it is still quite easy to get

$$
\frac{2}{d} \int d \boldsymbol{c}_{1} c_{1}^{2} \Lambda\left(\boldsymbol{c}_{1}\right) \delta \widetilde{F}_{1}\left(\boldsymbol{l}_{1}, \boldsymbol{c}_{1}, s\right)=-\delta \zeta_{0}(\boldsymbol{l}, s)+\zeta_{0} \delta \epsilon(\boldsymbol{l}, s)
$$

The expression of the fluctuating cooling rate $\delta \zeta_{0}$ is given in Eq. (67).

Also the calculation of the second moment of the noise term $\widetilde{S}_{\epsilon}$ is more cumbersome than for the other ones. Actually, it is the only one that does not vanish. The result is provided by Eq. (69) with

$$
\begin{aligned}
\Phi\left(\boldsymbol{c}_{1}, \boldsymbol{c}_{2}\right) \equiv & \frac{\pi^{\frac{d-1}{2}}}{\Gamma\left(\frac{d+5}{2}\right) d^{2}}\left\{\left[d+1-4 \alpha-(d+5) \alpha^{2}\right] C^{2} c_{12}^{3}+\frac{\left(1-\alpha^{2}\right)\left(d+5-2 \alpha^{2}\right)}{4} c_{12}^{5}\right. \\
& \left.+2(1+\alpha)(2 d+3-3 \alpha) c_{12}\left(\boldsymbol{C} \cdot \boldsymbol{c}_{12}\right)^{2}\right\}
\end{aligned}
$$

where $\boldsymbol{c}_{12} \equiv \boldsymbol{c}_{1}-\boldsymbol{c}_{2}$ and $\boldsymbol{C} \equiv\left(\boldsymbol{c}_{1}+\boldsymbol{c}_{2}\right) / 2$. 


\section{APPENDIX B: DERIVATION OF EQ. (105)}

The Fourier transform of Eq. (52) is

$$
\left\langle\widetilde{S}(\boldsymbol{k}, \boldsymbol{c}, s) \widetilde{S}\left(\boldsymbol{k}^{\prime}, \boldsymbol{c}^{\prime}, s^{\prime}\right)\right\rangle_{\mathrm{H}}=\delta_{\boldsymbol{k},-\boldsymbol{k}^{\prime}} \delta\left(s-s^{\prime}\right) \frac{\widetilde{V}^{2}}{N} \widetilde{\Gamma}\left(\boldsymbol{c}, \boldsymbol{c}^{\prime}\right) .
$$

where $\widetilde{V} \equiv V \lambda^{-d}, V$ being the volume of the system. Then, for any two components of $\boldsymbol{W}_{\perp}$, from Eq. (104) it is obtained that

$$
\begin{aligned}
\left\langle W_{\perp, i}(\boldsymbol{k}, s) W_{\perp, j}\left(\boldsymbol{k}^{\prime}, s^{\prime}\right)\right\rangle_{\mathrm{H}}= & \frac{\widetilde{V}^{2}}{N} \delta_{i j} \delta_{\boldsymbol{k}-\boldsymbol{k}^{\prime}} k^{2} \int_{0}^{s} d s_{1} \int_{0}^{s^{\prime}} d s_{1}^{\prime} \int d \boldsymbol{c} \int d \boldsymbol{c}^{\prime} c_{\|} c_{\perp, i} c_{\|}^{\prime} c_{\perp, j}^{\prime} \mathcal{U}\left(\boldsymbol{k}, \boldsymbol{c}, s_{1}\right) \\
& \times \mathcal{U}\left(-\boldsymbol{k}, \boldsymbol{c}^{\prime}, s_{1}^{\prime}\right) \delta\left(s-s_{1}-s^{\prime}+s_{1}^{\prime}\right) \mathcal{P}_{\perp} \mathcal{P}_{\perp}^{\prime} \widetilde{\Gamma}\left(\boldsymbol{c}, \boldsymbol{c}^{\prime}\right) .
\end{aligned}
$$

Here $\mathcal{P}^{\prime}$ is defined like $\mathcal{P}$, but acting on functions of the velocity $\boldsymbol{c}^{\prime}$, and $c_{\|}$denotes de component of $\boldsymbol{c}$ along $\boldsymbol{k}$. Now, it is taken into account that: i) Because calculations are restricted to the lowest order in $k$, which is $k^{2}$, the $k$ factors in the $\mathcal{U}$ operators can be neglected, i.e. the operator $L(\boldsymbol{k}, \boldsymbol{c})$ in Eq. (85) can be replaced by $\Lambda(\boldsymbol{c})$, and ii), Eq. (81) implies that, in particular, $\left\langle\chi c_{i} c_{j} \mid \xi_{\beta}\right\rangle=0 ; \beta=1, \ldots, d+2$, for $i \neq j$. Then, the projection operator $\mathcal{P}_{\perp}$ can be eliminated everywhere in Eq. (B2 $)$. Note that this can be only done after the operator $L(\boldsymbol{k}, \boldsymbol{c})$ has been replaced by $\Lambda(\boldsymbol{c})$. Then by carrying out the integral over $s_{1}$, it is obtained

$$
\begin{aligned}
\left\langle W_{\perp, i}(\boldsymbol{k}, s) W_{\perp, j}\left(\boldsymbol{k}^{\prime}, s^{\prime}\right)\right\rangle_{\mathrm{H}}= & \frac{\widetilde{V}^{2}}{N} \delta_{\boldsymbol{k},-\boldsymbol{k}^{\prime}} k^{2} \int d \boldsymbol{c} \int d \boldsymbol{c}^{\prime} c_{\|} c_{\perp, i} c_{\|}^{\prime} c_{\perp, j}^{\prime} e^{\left(s-s^{\prime}\right) \Lambda(\boldsymbol{c})} \\
& \times \int_{0}^{s^{\prime}} d s_{1}^{\prime} e^{s_{1}^{\prime}\left[\Lambda(\boldsymbol{c})+\Lambda\left(\boldsymbol{c}^{\prime}\right)\right]} \widetilde{\Gamma}\left(\boldsymbol{c}, \boldsymbol{c}^{\prime}\right),
\end{aligned}
$$

for $s>s^{\prime}$. Again, it is assumed that the HCS is linearly stable with respect to homogeneous perturbations for the system under consideration. Then, taking the limit $s^{\prime} \gg 1$, the above equation can be rewritten as

$$
\left\langle W_{\perp, i}(\boldsymbol{k}, s) W_{\perp, j}\left(\boldsymbol{k}^{\prime}, s^{\prime}\right)\right\rangle_{\mathrm{H}}=\frac{\widetilde{V}^{2}}{N} \delta_{\boldsymbol{k},-\boldsymbol{k}^{\prime}} k^{2} \int d \boldsymbol{c} \int d \boldsymbol{c}^{\prime} c_{\|} c_{\perp, i} c_{\|}^{\prime} c_{\perp, j}^{\prime} \times e^{\left(s-s^{\prime}\right) \Lambda(\boldsymbol{c})} \widetilde{\vartheta}_{\mathrm{HCS}}\left(\boldsymbol{c}, \boldsymbol{c}^{\prime}\right)
$$

The function $\widetilde{\vartheta}_{\mathrm{HCS}}\left(\boldsymbol{c}, \boldsymbol{c}^{\prime}\right)$ is the solution of the equation

$$
\left[\Lambda(\boldsymbol{c})+\Lambda\left(\boldsymbol{c}^{\prime}\right)\right] \widetilde{\vartheta}_{\mathrm{HCS}}\left(\boldsymbol{c}, \boldsymbol{c}^{\prime}\right)=-\widetilde{\Gamma}\left(\boldsymbol{c}, \boldsymbol{c}^{\prime}\right)
$$

i.e., (see Eq. (32)),

$$
\widetilde{\vartheta}_{\mathrm{HCS}}\left(\boldsymbol{c}, \boldsymbol{c}^{\prime}\right)=\int d \boldsymbol{l} \widetilde{h}\left(\boldsymbol{l}, \boldsymbol{c} ; \boldsymbol{c}^{\prime}\right) \text {. }
$$


To give account of the time dependence in Eq. (Bי $)$ introduce

$$
\widetilde{\psi}_{H C S}\left(\boldsymbol{c}, s ; \boldsymbol{c}^{\prime}\right)=\int d \boldsymbol{l} \widetilde{h}\left(\boldsymbol{l}, \boldsymbol{c}, s ; \boldsymbol{c}^{\prime}\right) .
$$

It obeys the equation (see Eq. (30) )

$$
\left[\frac{\partial}{\partial s}-\Lambda(\boldsymbol{c})\right] \widetilde{\psi}_{H C S}\left(\boldsymbol{c}, s ; \boldsymbol{c}^{\prime}\right)=0 .
$$

By writing Eq. (B4) in terms of $\widetilde{\psi}_{H C S}\left(\boldsymbol{c}, s ; \boldsymbol{c}^{\prime}\right)$, Eq. (105) follows after a trivial change in the notation, taking into account the isotropy of $\Lambda(\boldsymbol{c})$.

\section{APPENDIX C: SOME DETAILS OF THE CALCULATIONS DESCRIBED IN} SEC. VI

The explicit expression of the function $G(|s|)$ defined in Eq. (106) is

$$
G(|s|)=\int d \boldsymbol{c} \int d \boldsymbol{c}^{\prime} c_{x} c_{y} c_{x}^{\prime} c_{y}^{\prime} e^{s \Lambda(\boldsymbol{c})} \chi(c) \delta\left(\boldsymbol{c}-\boldsymbol{c}^{\prime}\right)=\int d \boldsymbol{c} c_{x} c_{y} e^{s \Lambda(\boldsymbol{c})} \chi(c) c_{x} c_{y} .
$$

The term involving the two-particle correlation function $g_{\mathrm{HCS}}$ in Eq. (109) has been neglected. The reason for this is that numerical simulations of dilute granular gases [33] has shown that it gives much smaller contributions than the term kept. Substitution of the above expression in Eq. (113) and integration over time $s$ leads to

$$
\widetilde{\eta}^{\prime}=2 \int d \boldsymbol{c} c_{x} c_{y} C_{x y}(\boldsymbol{c})
$$

where $C_{x y}(\boldsymbol{c})$ obeys the equation

$$
\left[\Lambda(\boldsymbol{c})+\frac{\zeta_{0}}{2}\right] C_{x y}(\boldsymbol{c})=-\chi(c) c_{x} c_{y}
$$

This equation will be now solved in the first Sonine approximation, i.e. by keeping only the first term of the expansion of the solution in Sonine polynomials [20], that due the isotropy of the operator $\Lambda(\boldsymbol{c})$ has the form

$$
C_{x y}(\boldsymbol{c})=A c_{x} c_{y} \varphi_{0}(c)
$$

where $\varphi_{0}$ is the Gaussian

$$
\varphi_{0}=\pi^{-d / 2} e^{-c^{2}} .
$$


To determine the constant $A$, Eq. (C3) is multiplied by $c_{x} c_{y}$ and integrated over $\boldsymbol{c}$, after replacing $C_{x y}(\boldsymbol{c})$ by its expression in Eq. (C4). Moreover, the velocity distribution of the HCS is also approximated by its expression in the first Sonine approximation, Eq. (22). From this point, the only remaining task is to evaluate several integrals. Although lengthy, the calculation is straightforward and similar to the one needed to compute the Navier-Stokes transport coefficients [30]. The result found for the constant $A$ reads

$$
A=\frac{1+a_{2}(\alpha)}{4 \widetilde{\nu}(\alpha)-\frac{3 \zeta_{0}}{2}}
$$

with $\widetilde{\nu}(\alpha)$ given by Eq. (97). Finally, Eq. (C4) is substituted in Eq. (C2) and the result (114) follows quite easily.

In order to derive an expression for the two-time correlation function of the transverse velocity, Eqs. (105) and (110) are employed. Taking $s>s^{\prime} \gg 1$,

$$
\begin{aligned}
\left\langle\delta \omega_{\perp, i}(\boldsymbol{k}, s) \delta \omega_{\perp, j}\left(\boldsymbol{k}^{\prime}, s^{\prime}\right)\right\rangle_{\mathrm{H}}= & \frac{\widetilde{V}^{2}}{N} k^{2} \delta_{i, j} \delta_{\boldsymbol{k},-\boldsymbol{k}^{\prime}}\left[\int_{-\infty}^{s^{\prime}} d s_{1} \int_{-\infty}^{s^{\prime}} d s_{1}^{\prime} e^{\lambda_{\perp}(k)\left(2 s^{\prime}-s_{1}-s_{1}^{\prime}\right)} G\left(\left|s_{1}-s_{1}^{\prime}\right|\right)\right. \\
& \left.+\int_{s^{\prime}}^{s} d s_{1} \int_{-\infty}^{s^{\prime}} d s_{1}^{\prime} e^{\lambda_{\perp}(k)\left(s+s^{\prime}-s_{1}-s_{1}^{\prime}\right)} G\left(\left|s_{1}-s_{1}^{\prime}\right|\right)\right] .
\end{aligned}
$$

The first integral on the right hand side is related with the coefficient $\widetilde{\eta}^{\prime}$ computed before,

$$
\int_{-\infty}^{s^{\prime}} d s_{1} \int_{-\infty}^{s^{\prime}} d s_{1}^{\prime} e^{\lambda_{\perp}(k)\left(2 s^{\prime}-s_{1}-s_{1}^{\prime}\right)} G\left(\left|s_{1}-s_{1}^{\prime}\right|\right)=-\frac{\widetilde{\eta}^{\prime}}{2 \lambda_{\perp}(k)} .
$$

The other integral in Eq. (C7) can be evaluated for instance by introducing the variables $g \equiv s_{1}-s_{1}^{\prime}$ and $S \equiv\left(s_{1}+s_{1}^{\prime}\right) / 2$. Then, the integral is decomposed into three. For large enough $s-s^{\prime}$, the only remaining one is

$$
\int_{0}^{\infty} d g G(|g|) \int_{s^{\prime}-\frac{g}{2}}^{s^{\prime}+\frac{g}{2}} d S e^{\lambda_{\perp}(k)\left(s+s^{\prime}-2 S\right)}=-\frac{\widetilde{\eta}_{1}}{2 \lambda_{\perp}(k)} e^{\lambda_{\perp}(k)\left(s-s^{\prime}\right)},
$$

with $\widetilde{\eta}_{1}$ given by Eq. (116). Use of Eqs. ([C8) and (C9) into Eq. ([C7) gives Eq. (115).

[1] A. Goldshtein and M. Shapiro, J. Fluid Mech. 282, 75 (1995).

[2] J.W. Dufty, J. Phys.: Condens. Matter 12, A47 (2000).

[3] I. Goldhirsch, Annu. Rev. Fluid Mech. 35, 267 (2003). 
[4] Granular Gas Dynamics, edited by T. Pöschel and N.V. Brilliantov (Spinger-Verlag, Berlin, 2003).

[5] N.V. Brilliantov and T. Pöschel, Kinetic Theory of Granular Gases; (Springer-Verlag, Berlin, 2004).

[6] H.M. Jaeger, S.R. Nagel, and R.P. Behringer, Rev. Mod. Phys. 68, 1259 (1996).

[7] C.S. Campbell, Ann. Rev. Fluid Mech. 22, 57 (1990).

[8] M. Bixon and R. Zwanzig, Phys. Rev. 187, 267 (1969).

[9] M. Bixon, J.R. Dorfman, and J.W. Dufty, J. Phys. Chem. 93, 7019 (1989).

[10] M.C. Marchetti and J.W. Dufty, in Nonequilibrium Fluid Behavior, edited by H. Harley (North-Holland, Amsterdam, 1983).

[11] M.H. Ernst and E.G.D. Cohen in Perspectives in Statistical Physics, edited by H.J. Raveché (North-Holland, Amsterdam 1981).

[12] M.H. Ernst and E.G.D. Cohen, J. Stat. Phys. 25, 153 (1981).

[13] J.J. Brey, M.I. García de Soria, P. Maynar, and M.J. Ruiz-Montero, Phys. Rev. E, 70, 011302 (2004).

[14] P. Visco, A. Puglisi, A. Barrat, F. van Wijland, and E. Trizac, EPJB 51, 377 (2006).

[15] A.M. Tremblay in Recent Developments in Nonequilibrium Thermodynamics, edited by J. Casas-Vázquez, D. Jou, and G. Lebon (Springer-Verlag, Berlin, 1984).

[16] F.L. Hinton, Phys. Fluids 13, 857 (1970).

[17] R. Fox and G.E. Uhlenbeck, Phys. Fluids 13, 1893 (1970).

[18] L.D. Landau and E.M. Lifschitz, Fluid Mechanics, (Pergamon Press, Oxford, 1966).

[19] R. Kubo, M. Toda, and N. Hashitsume, Statistical Physics II (Springer-Verlag, Berlin, 1985).

[20] P. Résibois and M. de Leener, Classical Kinetic Theory of Fluids (Wiley, New York, 1977).

[21] J. Keizer, Statistical Thermodynamics of Nonequilibrium Processes (Springer-Verlag, Berlin, 1987).

[22] J.J. Brey and J.W. Dufty, Phys. Rev. E 72, 011303 (2005).

[23] A. Puglisi, A. Baldassarri, and V. Loreto, Phys. Rev. E 66, 061305 (2002).

[24] A. Puglisi, A. Baldassarri, and A. Vulpiani, J. Stat. Mech.: Theory Exp. 2007 P08016.

[25] T.P.C. van Noije, M.H. Ernst, R. Brito, and J.A.G. Orza, Phys. Rev. Lett. 79, 411 (1997).

[26] P.K. Haff, J. Fluid Mech. 134, 401 (1983).

[27] T.P.C. van Noije and M. Ernst, Granular Matter 1, 57 (1998). 
[28] S.H. Noskowitz, O. Bar-Lev, D. Serero, and I. Goldhirsch, Europhys. Lett. 79, 600001 (2007).

[29] J.J. Brey, J.W. Dufty, and M.J. Ruiz-Montero in Granular Gas Dynamics, edited by T. Pöschel and N. Brilliantov (Springer-Verlag, Berlin 2003).

[30] J.J. Brey, J.W. Dufty, C.S. Kim, and A. Santos, Phys. Rev. E 58, 4638 (1998).

[31] J.J. Brey and D. Cubero, in Granular Gases, edited by T. Pöschel and S. Luding (SpringerVerlag, Berlin, 2001).

[32] J.W. Dufty and J.J. Brey, J. Stat. Phys. 109, 433 (2002).

[33] J.J. Brey and M.J. Ruiz-Montero, Phys. Rev. E 70, 051301 (2004).

[34] J.J. Brey, M.I. García de Soria, and P. Maynar, to be published. 